\title{
Abstracts from \\ Center to Advance Palliative Care National Seminar Palliative Care Everywhere: Bridging the Gaps

\author{
November 12-14, 2015 \\ San Antonio, TX
}

The topic areas for abstract submission include:

Palliative Care Delivery/Operations

- Innovative and emerging community-based palliative care models (e.g., home/ office/clinic/long-term care)

- Innovative hospital-based palliative care models

- Integration of palliative care services in cancer centers/dialysis centers, and other special care units

- Approaches to palliative care delivery in specific hospital settings (e.g., ICU, ED)

- Approaches to palliative care delivery for specific population types (e.g., heart failure/cancer/dementia/frailty) or in specific settings (ICU, ED)

- Innovative pediatric palliative care programs including combined adult/pediatric models

- Integrating palliative care services (e.g., across a health system/patient-centered medical home/assisted living/nursing homes)

- Successful development of pharmacy roles on a palliative care team

- Implementation of standardized interventions such as checklists and screening tools

- Successful approaches to conducting an effective needs assessment 


\section{Palliative Care Teams}

- Staffing the interdisciplinary team including staffing benchmarks and maximizing the roles of social workers/chaplains/nursing and medicine

- How teams work together to maximize team health and avoid burnout

- Demonstrating clinical and financial outcomes for team roles

\section{Communication}

- Communication best practices (e.g., teaching goals of care conversations/advance care planning/family meetings)

- Implementation of POLST/MOLST

\section{Education}

- Methods of training in primary/generalist palliative care

- Examples of primary palliative care education approaches to address the workforce shortage

- Targeting Education: Pain management and communication skills

\section{Data and Outcomes}

- Using technology to improve efficiency and outcomes

- Data demonstrating depth of palliative care integration in ACO/PCMH/bundling and other risk-bearing health care delivery frameworks

- Successful use of metrics in community or hospital settings

Financing and Payer Collaborations

- Successful payer/provider collaborations and partnerships

- Financing/benefit and payment models

Coalition and Advocacy

- Regional and National Palliative Care Initiatives (e.g., statewide coalitions/political advocacy)

Other

1. The FM Effect on EoL Decisions for AAs by Medical Surrogates

Abington Hospital, Jefferson Health

Abington, PA

Team/Contact Information:

Maria Doll Shaw, DNP

Clinical Nurse Manager, Palliative Care Service

Abington Hospital, Jefferson Health

517 S. Schuylkill Avenue

Abington, PA 19403

(215) 481-8713

mdoll-shaw@abingtonhealth.org

Topics: Communication: Communication best practices (e.g., teaching goals of care conversations/advance care planning/ family meetings)

\section{Description:}

A retrospective pilot study of 96 EMRs of incapacitated, hospitalized, AA suffering with life-limiting illnesses whose medical surrogates participated in a PCT-led family meeting to determine decisions for EoL care. Patient median age was 78 years, $72 \%$ female; $13.5 \%$ had a LW, and $69.2 \%$ were upheld by the surrogates. Of the surrogates, $45.8 \%$ were the adult daughters. The average LOS was 17 days. For those transitioned to comfort care following the PCT-led family meeting the average LOS was reduced to 10.9 days.

Conclusion: PCT-led FMs can be effective interventions for this patient population at EoL. Few in this sample had LWs, but if they did, they were generally upheld by the surrogates; and so they are fairly reliable as a tool to provide the care pathway patients prefer when they are unable to advocate for themselves. 
The burden of EoL decision making often falls to the adult daughter. Knowing in advance characteristics and priorities of meeting participants can assist PCTs as they prepare for these FMs. Finally, organizations as well as patients can benefit from a decreased LOS in the acute care setting because they transitioned to the comfort-focused care pathway.

AA, African American; EoL, end of life; FM, family meeting; LOS, length of hospital stay; LW, living will; PCT, palliative care team.

\section{Community Palliative Care Reduces Hospital Readmission}

Allina Health Hospice and Palliative Care

St. Paul, MN

Team/Contact Information:

Joe Amberg, MD

Medical Director Palliative Care

Allina Health Hospice and Palliative Care

1055 Westgate Drive, Suite 100

St. Paul, MN 55114

(612) 262-7693

Joseph.amberg@allina.com

Project Team Members:

Pat Peschman, DNP, Manager

Jennifer Trygg, Data Analyst, Home Care Services

Topics: Palliative Care Delivery/Operations: Innovative and emerging community-based palliative care models (e.g., home/ office/clinic/long-term care)

Description:

The Allina Health Community Palliative Care Team has provided service in the Twin Cities, MN, community for 8 years. The team is composed primarily of eight specially trained home health nurses who make home visits, with twice monthly IDT collaboration with MD, social worker, and spiritual care. The team successfully provides expert symptom relief and coordination of care for patients with complex illness who continue life-prolonging treatment, with high rates of referral to hospice when warranted by the patient's condition and goals of care. This year we are collecting data on hospital readmissions, with dramatic reductions measured for patients receiving palliative care. Patients assessed as higher risk and worse prognosis by OASIS screen showed the greatest benefit.

Results: For patients with temporary at-risk status with return to normal health, readmission rate is $12.1 \%$ with no PC, and $3.7 \%$ with PC. For patients at high risk and fragile status, readmission rate is $21 \%$ with no $\mathrm{PC}$, and $5.6 \%$ with PC. For patients in serious condition and with prognosis less than one year, readmission rate is $33.9 \%$ with no PC, and $2.6 \%$ with PC. This is the grand total for January to June 2015, and we continue rolling data, which can be updated at the time of poster presentation.

3. Inpatient Palliative Care Reduces Hospital Readmissions Allina Health Hospice and Palliative Care

St. Paul, MN

Team/Contact Information:

Joe Amberg, MD

Medical Director Palliative Care

Allina Health Hospice and Palliative Care

1055 Westgate Drive, Suite 100

St. Paul, MN 55114

(612) 262-7693

Joseph.amberg@allina.com
Project Team Members:

Jennifer Trygg, Data Analyst, Home Care Services

Topics: Data and Outcomes: Successful use of metrics in community or hospital settings

Description:

Allina Health Palliative Care staffs inpatient consult teams at four metro hospitals in the Twin Cities, MN, and one regional hospital in Shakopee. The teams are well established, providing expert symptom care, goals of care discussion, and end-of-life care for hospital patients with serious illness, working in close collaboration with hospitalists, intensivists, and other specialists. Three hospital teams (two new in the past year) also provide weekly outpatient clinic consultation and follow-up. One hospital provides training for the University of Minnesota HPM Fellowship. In 2014 the teams provided 4047 consults and 10,453 follow-up visits. For this study we reviewed hospital admissions in 2012 to 2013.

Results: We measured number of hospitalizations before and after inpatient palliative care intervention, for 2 years including January 2012 to December 2013. The grand total of measured patients is 7930, with an average of 2.67 hospitalizations before IPPC consult, and 0.82 hospitalizations after IPPC consult. Each of the five hospital teams shows statistically similar results. To address whether these patients were simply being seen at the end of life, further data on patients living 30, 90, 120, and 240 days after discharge are available, all showing similar reductions (e.g., 2.58 versus 1.28 hospitalizations for alive $>240$ days).

\section{Palliative Care Reduces Hospital Costs at End of Life} Allina Health Hospice and Palliative Care

St. Paul, MN

Team/Contact Information:

Joe Amberg, MD

Medical Director Palliative Care

Allina Health Hospice and Palliative Care

1055 Westgate Drive, Suite 100

St. Paul, MN 55114

(612) 262-7693

Joseph.amberg@allina.com

Project Team Members:

Lori Motschenbacher, Manager, System Financial Analysis

Topics: Palliative Care Teams: Demonstrating clinical and financial outcomes for team roles

Description:

Study information: Net hospital costs in 2011 were tabulated for patients who died in the hospital, with length of stay of 4 days or greater, and who received palliative care consultation at least 2 days prior to death. Costs were measured for the final 3 hospital days, with data specific to hospital, patient diagnosis, and allocation of costs. A comparison group are patients with the same inclusion criteria, but who did not receive palliative care consultation.

\begin{tabular}{llrcc}
\hline \multicolumn{2}{l}{ Savings per consult } & Net savings & Expense & Team size \\
\hline Abbott & $\$ 3,702$ & $\$ 685,000$ & $\$ 317,000$ & 7.3 \\
United & $\$ 1,721$ & $\$ 215,000$ & $\$ 210,000$ & 2.6 \\
Mercy & $\$ 1,935$ & $\$ 122,000$ & $\$ 104,000$ & 3.0 \\
Unity & $\$ 1,734$ & $\$ 110,000$ & $\$ 124,000$ & 1.6 \\
TOTAL & & $\$ 1,132,000$ & $\$ 775,000$ &
\end{tabular}


Results: The table below provides a summary for the four hospitals. The average cost for the final 3 hospital days was always greater for patients with no palliative care, regardless of diagnosis or hospital. There were a total of 475 palliative care consults provided meeting study inclusion criteria, compared with 239 patients who were not seen by palliative care. This study group represents only $13 \%$ of total consults seen, and demonstrates cost avoidance that exceeds total program costs.

\section{Patient and Caregiver Experience with Care}

Allina Health

Minneapolis, MN

Team/Contact Information:

Laura Sutter, MPH

Research Coordinator

Allina Health

2925 Chicago Avenue, MR 10039

Minneapolis, MN 55407

(612) 262-0534

laura.sutter@allina.com

Project Team Members:

Nathan Shippee, MS, PhD

Tetyana Shippee, MS, PhD

Karl Fernstrom, MPH

Cindy Cain, MA PhD

Alissa Jones, MA

Heather Britt, MPH, PhD

Topics: Palliative Care Delivery/Operations: Approaches to palliative care delivery for specific population types (e.g., heart failure/cancer/dementia/frailty)

Description:

Aim: Assess experience in LifeCourse, a late-life supportive care intervention, using a new experience tool for patients and caregivers.

Data and analysis: Intervention sample with baseline and 6-month data included 373 heart failure, cancer, and dementia patients and 434 caregivers. Usual care included 201 patients and 163 caregivers. Experience was assessed quantitatively through quarterly administration of a 30-item tool built to study guiding principles as sub-domains, and overall ratings for care and support. Qualitative data came from patient and caregiver interviews, and was used to better understand patterns.

Results: Intervention patients trended toward greater improvements in all sub-domains and overall care and support ratings, compared with those in usual care. Findings were more mixed for caregivers.

Conclusions: Experience scores showed more positive trends for LifeCourse than for comparison patients (although without significance in most domains by 6 months.) The patterns were slightly more consistent for patients than caregivers, reflecting potential benefits of LifeCourse and challenges to engaging caregivers. We look to perform longer follow-up and other components of assessment. Care delivery and experience tools oriented toward ongoing relationships and later-life care for complex patients may allow for meaningful assessment and better understanding of goals for integrating and streamlining care.

\author{
6. Patient and Caregiver Quality of Life \\ Allina Health \\ Minneapolis, MN \\ Team/Contact Information: \\ Laura Sutter, $M P H$ \\ Research Coordinator \\ Allina Health \\ 2925 Chicago Avenue, MR 10039
}

Minneapolis, MN 55407

(612) 262-0534

laura.sutter@allina.com

Project Team Members:

Nathan Shippee, $M S, P h D$

Tetyana Shippee, $M S, P h D$

Karl Fernstrom, $M P H$

Cindy Cain, MA PhD

Alissa Jones, $M A$

Heather Britt, $M P H, P h D$

Topics: Palliative Care Delivery/Operations: Approaches to palliative care delivery for specific population types (e.g., heart failure/cancer/dementia/frailty)

Description:

Question: Does participation in LifeCourse provide better quality of life (QOL) for late-life patients with chronic conditions and their caregivers compared with usual care controls?

Data: Intervention sample with baseline and 6-month data included 373 heart failure, cancer, and dementia patients and 434 caregivers. Usual care included 201 patients and 163 caregivers.

Measures: QOL was measured quarterly. Patient QOL was measured using the FACIT-PAL, and caregiver QOL was measured using the PROMIS-29. We also collected qualitative interviews.

Results: Stability in QOL for LifeCourse patients versus negative change for usual care, and improvements for LifeCourse patients in emotional and palliative care subscales. Interviews provided examples of a positive impact across subscales. Marginal positive scores for caregivers enrolled in LifeCourse compared with usual care controls.

Conclusions: LifeCourse helped maintain and even improve some QOL domains. This is notable because late-life patients face declining health and caregivers often have high caregiving burden. LifeCourse assessments of patient and caregiver QOL meet a need for information about patient and family functioning and the role of health care delivery in whole-person care. Whole-person supportive care interventions are a promising approach for patients and caregivers affected by complex chronic illness at the end of life.

\section{Late Life Care Approach Decreased Utilization and TCOC}

Allina Health

Minneapolis, MN

Team/Contact Information:

Laura Sutter, $M P H$

Research Coordinator

Allina Health

2925 Chicago Avenue, MR 10039

Minneapolis, MN 55407

(612) 262-0534

laura.sutter@allina.com

Project Team Members:

Karl Fernstrom, MPH

Rebecca Prenevost, $M P H, P h D$

TC Tong

Heather Britt, MPH, PhD

Topics: Palliative Care Delivery/Operations: Approaches to palliative care delivery for specific population types (e.g., heart failure/cancer/dementia/frailty)

Description:

Aim: Evaluate the effects of LifeCourse, a late-life care approach, on health care utilization and total cost of care in a large health care delivery system. 
Data: 373 intervention and 201 usual care patients between October 2012 and April 2015. A subset of patients with total cost of care claims data available was selected for cost analysis, with controls selected from the health care system's patient population and matched using propensity scores.

Results: LifeCourse patients in their last 6 months of life had half as many inpatient days as usual care controls. No significant difference in the number of ED visits. The proportion of advance directives increased significantly in the follow-up period compared with usual care. LifeCourse patients experienced higher inpatient and community-based palliative care utilization. The proportion of hospice enrollment prior to death was higher in the intervention group than the usual care group, the health care system in general, and the 2013 NHPCO national estimate. Also, average per patient per month total expenditures decreased more in the intervention group.

Conclusions: Early signals indicate that using a whole person approach and allowing patients to voice preferences and take part in care decisions may benefit utilization and total cost of care.

\section{Palliative Care through a Dementia Lens}

Alzheimer's Association, New York City Chapter

New York, NY

Team/Contact Information:

Ann Wyatt, MSW

Alzheimer's Association, New York City Chapter

360 Lexington Avenue, $4^{\text {th }}$ Floor

New York, NY 10017

(646) 744-2963

awyatt@alznyc.org

Project Team Members:

Tena Alonzo, MA

Natasha Bryant, MS

Edward Cisek, PhD

Mark Heisler, PharmD

Robert Hess, PhD

Mark Kator, CEO

Jed Levine, MA

Tony Lewis, CEO

Carol O. Long, PhD

Karen Mitchell, BSN

Robyn I. Stone, DrPH

Audrey Weiner, CEO

Ann Wyatt, MSW

Topics: Palliative Care Delivery/Operations: Approaches to palliative care delivery for specific population types (e.g., heart failure/cancer/dementia/frailty)

\section{Description:}

Cambia Health Solutions has implemented a health planbased serious illness services program for the 2.2 million members we serve, with an approach focused on respecting the patient's wishes, from diagnosis to completion of life. This program puts the patient at the center while remaining inclusive of the entire health care community. New health care services, benefits, training, and education have been created to ensure the entire health care system is engaged in putting the patient and their loved ones at the centersupporting the individual and family in their medical, psychosocial, behavioral, and spiritual needs. In addition, we provide necessary support and resources to caregivers and loved ones dealing with the serious illness of a loved one or in need of grief or bereavement counseling. Cambia has focused its efforts in integrating five main areas: benefit expansion, specialized care management, community and member en- gagement, provider reimbursement and training, and caregiver support. This presentation will provide our framework and implementation of this program, providing a structure for other payers to use in development of palliative care initiatives. We will represent initiatives within and outside of our Accountable Care Organization agreements. Example metrics and baseline statistics will also be presented.

\section{Cultivating a Culture of Primary Palliative Care \\ Baptist Health}

Jacksonville, FL

Team/Contact Information:

Tasha M. Schoppee, MSN

Baptist Health

800 Prudential Drive

Jacksonville, FL, 32207

(904) 202-3409

Tasha.Schoppee@bmcjax.com

Topics: Education: Methods of training in primary/generalist palliative care

Description:

Whether in the arena of acute care, the clinic setting, or in the home, comprehensive palliative care requires collaboration among both specialist and generalist clinicians. Developing the infrastructure to support such a cooperative practice requires strategic communications and intentional education across the disciplines. Successful development of such a culture necessitates intentionally targeting, and inviting in, many who have not previously been passionate about palliative care. They must have the opportunity to meet on mutual ground. One hospital system has found establishment of an interprofessional palliative care council to be the environment in which such collaboration can be nurtured. Additionally, there is great value in developing this foundation prior to the building of a formal palliative care program. Therefore, an interdisciplinary council is a powerful tool for both those organizations with mature palliative programs and those who are still in the planning stages.

10. An Assessment of Palliative Care Beliefs and Knowledge

Baptist Health of South Florida

Miami, FL

Team/Contact Information:

Yvonne Patten

Baptist Health of South Florida

8900 North Kendall Drive

Miami, FL 33176

(786) 596-0092

yvonnep@baptisthealth.net

Project Team Members:

Maria M. Ojeda, ARNP, MSN, MPH, DNP, PHD

Carolyn L. Lindgren, PhD, RN

Topics: Education: Examples of primary palliative care education approaches to address the workforce shortage

\section{Description:}

This study evaluated the level of perceived competency and knowledge regarding palliative care among health care personnel at a seven-hospital not-for-profit health care system; and determined if there was a significant difference in perceived competency and knowledge, between health care personnel who participated in an intercultural palliative care/end-of-life training program and those who did not. 
Using a pre-experimental static-group comparison design, a non-randomized sample of health care personnel were administered a demographic questionnaire to assess perceived competence (End-of-Life Professional Caregiver Survey [EPCS]) and a knowledge assessment (Palliative Care Survey [PCS]). The results revealed a significant negative association between perceived competency and knowledge scores, (Spearman's rho $=-0.380, n=330, p=0.000$ ). Those who did not take the course perceived themselves more competent when compared with those who took the course (Mann-Whitney $U=21,332$, $\mathrm{z}=4.827, p=0.000$ ). Participants who took the training course scored significantly higher on knowledge than those who did not (Mann-Whitney $U=10,257.00, \mathrm{z}=-3.797, p=0.000$ ). This study supports the need for ongoing education to increase knowledge of palliative care among health care personnel and further exploration of the underlying reason for the negative association between perceived competency and knowledge.

11. Stand By You: An Interprofessional Compassionate Service

Baptist Health of South Florida

Miami, FL

Team/Contact Information:

Rose M. Allen, DNP

Baptist Health of South Florida

8900 North Kendall Drive

Miami, FL 33176

(786) 596-0092

rosea@baptisthealth.net

Project Team Members:

Rose Allen, DNP, MSM/HM, RN, CHPN

Chloe Edinger, BS, MS3

Renato Santos, MDiv, BCC, LMHC

Arlenna Williams, MSW, MBA

Sarita Khanal, ARNP, RN

Carol Slinger-Howell, MSN, RN, CNML

Topics: Palliative Care Delivery/Operations: Approaches to palliative care delivery for specific population types (e.g., heart failure/cancer/dementia/frailty)

\section{Description:}

This poster will outline the collaborative efforts between Herbert Wertheim College of Medicine and Baptist Health South Florida to develop an interprofessional compassionate volunteer service for patients at the end of life, including the program's integration across the health system and the outcomes.

The Stand By You (SBY) Volunteer service was developed in 2012 and initially offered at one of the seven hospitals within the system. It is based on the No One Dies Alone (NODA) program started by Sandra Clarke, CCRN, in 2001, with the goal to provide companionship and support for dying individuals, so they don't die alone. The SBY Volunteer program is a unique service where companionship and support is provided by first- and second-year medical student volunteers. Since 2014, the program has been expanded to include hospital volunteers of nurses, social workers, board members, and other community volunteers, who collaborate with the medical students to provide services at our hospitals within the health system.

So far, 99 volunteers have been trained (53 medical students, 22 employees, 24 community volunteers) and 16 patients to date at Baptist Health have received services by these volunteers. Satisfaction of the SBY service is measured by survey feedback from staff and family members, if available.
12. Examining Use of Advance Directives in Oncology Patients

Baptist Hospital, Inc.

Penscola, FL

Team/Contact Information:

Kelly C. Markham, MSW

Palliative Care Coordinator

Baptist Hospital, Inc.

1000 West Moreno Street

Pensacola, FL 32501

(850) 469-3739

kelly.markham@bhcpns.org

Topics: Palliative Care Delivery/Operations: Approaches to palliative care delivery for specific population types (e.g., heart failure/cancer/dementia/frailty)

\section{Description:}

In our cancer center, a retrospective chart review was conducted of stage III and IV lung cancer patients, examining the use of advance directives. The results revealed that only $57 \%$ of those patients had completed an advance directive. In almost one-quarter of cases, the chart was silent about documented directives. This prompted our team to seek more information about lack of advance care planning among our patients with this serious illness. Several possibilities were discussed: lack of initiation of discussion by physician and/or staff, patient resistance, or other reasons. To assess these barriers, a survey was compiled to inquire about roadblocks. Currently, it is being used in our radiation oncology clinic. At this time, 26 patients have completed the survey. Initial responses indicate that most patients "just never got around to doing it." The survey will continue to be utilized to obtain more information about barriers. Results of this survey, when concluded, will direct our cancer center's efforts to increase the understanding and completion of advance directives among our patients. Information already gained during these two projects has led us to expand to plan a system-wide needs assessment about the advance care planning process in our organization. This will be initiated in the spring of 2016 .

\section{CARE: A Model for Doctor-Surrogate Decision Making}

Batson Children's Hospital

University of Mississippi Medical Center

Jackson, MS

Team/Contact Information:

Charles Christian Paine, II, MD

Batson Children's Hospital

University of Mississippi Medical Center

402 Hemlock Drive

Flowood, MS 39232

(601) 842-0930

cpaine@umc.edu

Topics: Communication best practices (e.g., teaching goals of care conversations/advance care planning/family meetings)

Description:

Much has been written about the importance of the "DoctorPatient" relationship and the obligations it demands. A majority of attention has been paid to the various steps, checklists, and algorithms that providers must follow to insure that they allow patients an autonomous voice. Interestingly, although the importance of values such as trust, compassion, and respect are mentioned as vital to this relationship, less attention is given to how to instill and maintain these in practice. Further, although emotion is mentioned as a component of many of these models, it is mostly referenced as merely a reaction to be allowed and affirmed. Finally, the role of family or surrogates in the 
decision-making relationship is often missed. The CARE Method is an analytical tool to help providers, patients, and families better understand each other's motivations and attitudes. The method examines the means and content of communication, the affect or mood of those communications, the context and reasoning of each party, and the effect and conclusions of each interaction. In this way, one can achieve a better understanding of the emotional roots of a decision and thus have a positive effect on building and sustaining a therapeutic doctor-patient-surrogate relationship.

\section{Enhancing Care at End of Life through Transition to Hospice}

Baylor All Saints Medical Center at Fort Worth

Arlington, TX

Team/Contact Information:

Sommer Smith, MSW

Director, Comprehensive Care Management, Supportive and Palliative Carer

Baylor All Saints Medical Center at Fort Worth

6805 Glen Eagle Drive

Arlington, TX 76001

sommerp@baylorhealth.edu

Project Team Members:

Shawnta Pittman-Hobbs, MD

Sommer Smith, LMSW, ACM

Ellen Pitcher, RN, MSN, MBA NEA-BC FACHE

Leslie Phillips

Kimberly Montgomery, MSN, ACNS-BC, CCRN-CMC

Danielle Reed, LMSW, ACHP-SW

Chuck Wallace, MDiv, MA, BCC

Terry Boyd, RRT, RCP

April Jones, BSN, RN, CCTN

Diana Stone, MSN, RN

Topics: Palliative Care Delivery/Operations: Innovative hospital-based palliative care models

\section{Description:}

Patients who were at the end of life and required planned withdrawal of treatment often had withdrawal take place in the ICU and not in the optimal environment for grieving or allowing them access to the specialized knowledge and support services provided by hospice at end of life. The importance of this need is emphasized in a study in the Journal of Clinical Oncology (Wright, 2010) stating that among caregivers, $21 \%$ of those whose loved ones died in the ICU or hospital developed PTSD 6 months later compared with $4.4 \%$ of those whose loved ones died at home with hospice. Baylor All Saints Medical Center (BASMC) developed a process for critical care patients to transfer to a more home-like environment of the VITAS Hospice Inpatient Unit (located on the BASMC campus) for withdrawal of life-sustaining treatments. ICU nursing staff was provided with education about the benefits to hospice and processes were put into place that allowed for nursing to be a part of their patient's transfer to hospice. Improvements resulted in an increase of nursing staff understanding of hospice benefits and baseline of $0 \%$ of patients/families agreeing to transfer to hospice for planned withdrawal of life-sustaining treatment to $70 \%+$ agreeing.

\section{ESAS to Improve Symptomatology Management/ Communication}

Baylor St. Luke's Medical Center

Houston, TX

Team/Contact Information:

Eyitayo O. Odeniyi

Advanced Practice Clinician, Palliative Care Program
Baylor St. Luke's Medical Center

8835 Aspen Meadow Drive

Houston, TX 77071

(832) 355-5158

eodeniyi@stlukeshealth.org

Topics: Palliative Care Delivery/Operations: Implementation of standardized interventions such as checklists and screening tools Description:

As the cost of health care continues to rise and the American public clamors for improved safety and quality of health care, the Center for Medicare and Medicaid Services has linked patient's evaluation of providers with reimbursement for services. Specific expectations and benchmark scores outlined, and if unmet, providers will be financially penalized. Therefore, hospitals across the nation are implementing efforts to improve patient and family satisfaction.

The aim of this project is to answer the following questions. (1) Does the use of the Edmonton Symptom Assessment System improve registered nurses' knowledge of symptom assessment and management of patients with advanced and chronic diseases in an acute care hospital? (2) Will the implementation of the ESAS improve patients' and families' perception of communication with health care practitioners?

16. Living with Dying in the PICU: A Nursing Perspective Bloomsburg University

Bloomsburg, PA

Team/Contact Information:

Debbie Stayer, PhD

Assistant Professor

Bloomsburg University

7 Cotswold Street

Danville, PA 17821

(570) 275-9454

dstayer@bloomu.edu

Topics: Palliative Care Delivery/Operations: Approaches to palliative care delivery in specific hospital settings (e.g., ICU, ED)

Description:

The purpose of this study was to explore the experiences of pediatric ICU nurses who provide palliative care to children with life-threatening or life-limiting illnesses and their families and to obtain an increased understanding of the meaning and interpretation of their experience. Exploring these experiences may reveal the essence of the phenomenon as well as an in-depth understanding of the contextual factors associated with the experience.

17. Rapid Palliative Care in ED Improves Care for Dementia

Bridgeport Hospital-Yale New Haven Health System

Bridgeport, CT

Team/Contact Information:

Kristen Edwards, MD

Chief of Palliative Care

Bridgeport Hospital-Yale New Haven Health System

New Canaan, CT 06840

(203) 337-8558

Kristen.Edwards@bpthosp.org

Project Team Members:

Vivian Argento, MD, Executive Director Geriatrics and Palliative Care (BH)

Gina Calder, FACHE, Executive Director of Clinical Services (BH)

Jennifer Kapo, MD, Chief of Palliative Care (YNHH)

Donna Coletti, MD, Chief of Palliative Care (GH) 
Kristin Edwards, MD, Chief of Palliative Care (BH) Rockman Ferrigno, MD, Chair, Department of Emergency Medicine (BH)

Karen Jubanyik, MD, Emergency Medicine (YNHH)

Ryan O'Connell, MD, VP Performance and Risk Management (BH)

Dominik Trzepacz, Finance Analyst

Cheryl Brophy, EPIC, Support

Topics: Palliative Care Delivery/Operations: Approaches to palliative care delivery in specific hospital settings (e.g., ICU, ED) Description:

Rapid palliative care assessments were done according to usual care by geriatric nurse practitioners and outcomes compared with those who met criteria for consultation based on a computer-based trigger for severity of illness and diagnosis of advanced dementia. Those who received consultation had a reduced rate of Quality Value Indicators (QVIs) compared with those who would have based on trigger criteria (7\% versus $9 \%$ ). For patients who had a QVI during their hospitalization, those seen by the consulting team had a reduction in direct cost per case of $\$ 12,058$ (\$7520 versus $\$ 19,578$ ), and a reduced length of stay by 8.44 days ( 9.67 days versus 18.11 days). In the group that did not have a QVI during hospitalization, cost reduction was also seen but not as significant (\$269; \$6267 versus \$6536) despite a slight ( 0.5 day) increase in ALOS (8.89 days versus 8.37 days). Consultation in the ED by geriatric trained APRNs lead to improved reduction in QVIs, and reduced cost and length of stay when QVIs occurred.

18. Using Case Conflict to Improve Consulting Relationships

Bridgeport Hospital-Yale New Haven Health System

Bridgeport, CT

Team/Contact Information:

Kristin Edwards, MD

Chief of Palliative Care

Bridgeport Hospital-Yale New Haven Health System

New Canaan, CT 06840

(203) 337-8558

Kristin.edwards@bpthosp.org

Project Team Members:

Kristin Edwards, MD, Chief of Palliative Care (BH)

Jennifer Healy, RN, ICU Nurse Educator (BH)

John-Paul Ayala, MD

Laura Ritter, RN, ICU Staff Nurse

Topics: Communication: Communication best practices (e.g., teaching goals of care conversations/advance care planning/ family meetings)

Description:

A clinical case in the intensive care unit generated conflict about how to manage acute urinary retention in a dying patient whose goals of care were ambiguous. Initial communication around this conflict created potential for misunderstanding and impaired relationships between consulting team, nursing staff, and referring team. We took a collaborative approach to resolving the conflict and considered the issues at hand, which included: staff discomfort with ambiguous goals of care, role of the consulting team in acute problem management, consultation etiquette, and conflict resolution for triangled relationships. Utilizing a systematic approach to the conflict, all involved parties were able to resolve the conflict and consequently strengthened their relationships with each other, generating further consults in the very same week. The case presented an example of how to use this collab- orative approach to best grow the consultation service, as well as meet the staff needs in situations of moral distress.

\section{Disseminating End-of-Life Nursing Education} throughout a Hospital System

Bryn Mawr Hospital, Main Line Health

Bryn, PA

Team/Contact Information:

Kelli M. Anspach, BSN, RN-BC

Bryn Mawr Hospital, Main Line Health

130 S. Bryn Mawr Avenue

Bryn, PA 19010

(215) 350-2108

anspachk@mlhs.org

\section{Project Team Members:}

Laura Bruce, $M E d, B S N, R N-B C, O C N$

Topics: Education: Methods of training in primary/generalist palliative care

\section{Description:}

Two nurse educators working to disseminate end-of-life education for nurses in a four-hospital acute-care health system. Goals are to enlist nurses' support to increase palliative care utilization, to strengthen knowledge, competence, and confidence in end-of-life care, and ultimately improve the patient and family experience. A proposal was created to garner leadership support using needs assessment statistics and linking learning objectives to the system's strategic plan and forces of magnetism. Potential barriers, plans to overcome obstacles, and the logistics of providing consistent education were discussed. Target units were identified for year-one goals. Grant money was awarded through a community partnership and supplemented by a family donation. Additional community resources were employed by incorporating home care partners and using local experts as speakers. Impact evaluation of the program, IRB exempt, includes a pre- and post-self-efficacy survey using the Palliative Care Self Efficacy Scale, with two qualitative questions and ongoing evaluation of HCAHPS scores. In order to sustain support for education HCAHPS scores of target units will be followed to determine if there is a relationship between attendance of the course with higher patient satisfaction scores.

\section{Organizations' Cultural Readiness for Palliative Care} Cancer Treatment Center of America Philadelphia, PA

\section{Team/Contact Information:}

Marie Decker, MSN, RN, AOCN, NE-BC, HN-BC

Supportive \& Palliative Care Nurse Manager

Cancer Treatment Center of America

326 Edgewood Avenue

(215) 537-7059

\section{Horsham, PA 19044}

marie.decker@ctca-hope.com

Topics: Palliative Care Delivery/Operations: Integration of palliative care services in cancer centers/dialysis centers and other special care units

\section{Description:}

NIH and JACHO are recommending the development palliative care as a supplementary service for patients. Also, oncologists are searching for practical solutions to holistically care for their oncology patients. Usually, these patients have latent needs to address; for instance, their continuum of care questions while transitioning from curative to non-curative treatment planning. One cancer center's solution was to develop, and 
integrate a palliative service program to engage the regulatory recommendations, and the patients' latent needs.

For two years, this center adopted the Center to Advance Palliative Care's business plan for facility readiness, need, and ability to create a palliative care service. The cultural "readiness" was defined by the center's patient-centric healing culture in conjunction with palliative care's positive attributes. The "need" spring-boarded from the integrative team's collaborative discussions with administrators. The center's "ability to create" became shared outcomes. The "results" were a board certified palliative medical director, a nurse manager, and 170 referred oncology patients. Currently, an interdisciplinary committee has finalized a gap analysis for the future needs of a $24 / 7$ program goal. The question for other organizations interested in palliative care is: "Do you understand critical success factors, and complex interdependencies before moving too far and too fast?"

\section{A PC Team's Journey toward Discovering Their Own} GOC

Carolinas HealthCare System

Carolinas Palliative Care \& Hospice Group

Charlotte, NC

Team/Contact Information:

Erica Frechman, MSN, AGPCNP-BC, ACHPN

Carolinas HealthCare System

Carolinas Palliative Care \& Hospice Group

3249 Basalt Place

Davidson, NC 28036

erica.frechman@carolinashealthcare.org

Project Team Members:

Kim Shellenberger, MSSW, LCSW

Topics: Palliative Care Teams: How teams work together to maximize team health and avoid burnout

Description:

Burnout became an imminent concern for an interdisciplinary palliative care team in a large hospital. The critical events of excessive workload and leadership/staffing changes impacted self-care strategies and compromised the sustained ability to care for seriously ill patients and families. This is one team's journey to pause, refocus, and take back ownership of their team's structure, team-wellness, and scope of services.

The team lead nurse practitioner and social worker designed a retreat to promote an innovative team culture encouraging open communication, analysis of work/life balance, professional gratification, and strategic planning. A creative and unique way to address communication, structure, and team health called "Wall of Words" was developed. This interactive exercise fostered ongoing conversations about matters the team had previously neglected.

This initial retreat began the journey to a revision of workflow processes, development of a scope of services document, and creation of a team-wellness strategy that prioritized team sustainability and a reinvigoration of team identity. This initiative can be a starting platform for other palliative care teams. Outcomes measured through pre-/post-retreat surveys showed improvement in assessment of team function and work/life balance. Ongoing events and retreats will prioritize this team's continued journey toward their goals of care.

\author{
22. Evaluating the State of Palliative Care in Colorado \\ Center for Improving Value in Health Care \\ Denver, CO \\ Team/Contact Information: \\ Kristin M. Paulson \\ Director of Health Care Programs
}

Center for Improving Value in Health Care

950 S. Cherry Street, \#208

Denver, CO 80246

(720) 242-7048

kpaulson@civhc.org

Topics: Palliative Care Delivery/Operations: Successful approaches to conducting an effective needs assessment

Description:

In 2013, the Center for Improving Value in Health Care commissioned a study to look at the extent and depth of palliative care provision across Colorado. The goal of the study was to evaluate: 1) the availability of hospital and community-based palliative care and 2) what services are provided as part of these palliative care programs. At the end of the study we compared the results with a similar study conducted in 2008 . The results will be used to drive payment reforms and to inform policy changes to better support provision of and access to palliative care.

The results showed a significant increase in the number of palliative care consults provided, but little to no increase in the number of providers or providing facilities. Providing facilities used team-based care in a large number of cases, though reimbursement issues mean that hospital-based programs can provide more robust team-based care than community-based programs. The distribution of facilities and programs also emphasized a severe lack of access to services in the rural areas of the state. Identified challenges to expanded provision of services included reimbursement, workforce, cultural misunderstanding, and access. We are working with partners to develop programs to address these challenges.

\section{Utilizing the EHR to Advance Palliative Care}

Cerner Corporation

Kansas City, MO

Team/Contact Information:

Hannah Luetke-Stahlman, MPA

Strategist

Cerner Corporation

8010 Ward Parkway Plaza

Kansas City, MO 64114

(816) 835-2107

Hannah.Luetke-Stahlman@Cerner.com

Project Team Members:

Marsha Laird-Maddox, Sr. Engagement Leader

Justin Kimbrell, Strategist Program Design Development

Topics: Data and Outcomes: Using technology to improve efficiency and outcomes

Description:

Cerner Corporation recognizes a need within the industry to electronically identify, engage, and manage palliative care patients so that care teams and providers can deliver the highest quality of care at the lowest cost. The program understands the multi-disciplinary approach to palliative medicine, providing clinicians with a holistic plan of care centered on individual patient and family goals. It is Cerner's intent to focus on quality of life for palliative care patients while generating the required electronic documentation, communication, data, and analytic tools to assist providers in accomplishing this objective.

Utilizing Cerner's new Palliative Care electronic solution, Ascension Health is partnering with the University of Pennsylvania to undertake an NIH-funded clinical trial launching October 2015. The innovative trial will be conducted at 11 Ascension Health hospitals over a 3-year study period and will provide the first experimental evidence of the effectiveness of inpatient palliative care consultative services in real-world settings. 
Cerner is also committed to collaborating with industry organizations and leaders as guidelines and metrics are developed. It is imperative that our solution fits within industry requirements and assists programs in meeting Joint Commission certification as well as national registry databases.

24. Educating the Interdisciplinary Team about Palliative Care: An Evidence-Based Project

Chapters Health System

Tampa, FL

Team/Contact Information:

Isabel Bales, DNP, ARNP

Nurse Practitioner

Chapters Health System

5100 Burchette Road \#103

Tampa, FL 33647

(309) 338-2369

www.chaptersheatlhsystem.org

Topics: Other

This quantitative study was to determine whether presenting educational content on palliative care to the hospital patient care staff would increase the number and timeliness of palliative care consults. The palliative care education was also evaluated by those who attended the presentation.

\section{Description:}

An educational project was implemented at St. Joseph's Hospital North (SJN), a small community-based hospital with 139 beds in Tampa, FL, in December 2013. The Stetler Model of Evidence-Based Practice was used to guide the development and implementation of this program. The curriculum content was presented to 19 staff members and addressed the following topics: differences between PC, hospice, and comfort care; appropriate timing and indications for PC consultation; benefits of PC for the patient, hospital, and hospital patient care staff; symptom management; cultural, spiritual, and ethical aspects of PC; advanced care planning and the family meeting. Survey Monkey was utilized to evaluate the PC education for those who attended the program. PC consults completed from January 2014 through May 2014 were compared with consults completed for 5 months prior to January 2014. In addition, in order to assess for seasonality, those consults completed in January 2014 through May 2014 were also compared with those completed in the same months one year prior. The Wilcoxon test was used to determine statistical differences in the number of consults before and after the intervention. The timeliness of PC consults was tested by evaluating the length of stay prior to the consult to the length of stay after the consult for 2 months before and after the educational intervention.

\section{Proving the Financial Benefit-Inpatient Palliative} Care

Community Health Network

Indianapolis, IN

Team/Contact Information:

Stacey E. Sharp, MBA

Life's Journey Program Director

Community Health Network

9894 E. 121st Street

Indianapolis, IN 46037

(317) 621-4631

ssharp3@ecommunity.com

Topics: Financing and Payer Collaborations: Financing/benefit and payment models

\section{Description:}

This poster session focus is based on proving the financial benefit of health systems funding palliative care consult teams within the walls of their hospitals. Community Health Network's Palliative Care Inpatient Consultation Team, Life's Journey: Choices in Care, has seen a $299.7 \%$ growth since the start of the program in 2006 through year-end 2014. Since 2013, the program has grown by $45.6 \%$ with a significant improvement in productive hours as well as proving what evidencedbased literature has shown ... that early palliative intervention equates to substantial cost savings.

During our poster session, we will also demonstrate the benefit around common barriers of supporting and implementing a palliative care program including the need for health systems to invest in a trained workforce to ensure sufficient numbers of specialists are available to both teach all clinicians the fundamentals in an integrative model as well as to directly provide high-quality palliative care for the highest-risk and most complex patients.

\section{Integrating Pharmacists into a Palliative Medicine} Team at a Community Hospital

Community Hospital of the Monterey Peninsula

Monterey, CA

Team/Contact Information:

Dharma Naidu, PHARMD

Clinical Pharmacist

Community Hospital of the Monterey Peninsula

22997 Espada Drive

Salinas, CA 93908

dharma.naidu@chomp.org

Project Team Members:

Dharma Naidu, PharmD, BCOP

Christine Short, RN

Ethan Howe, NP

Kacie Boyce, $R N$

Kim Jones, PharmD

John Juster, MSW

Chris Williams, MDiv

John Hausdorff, MD, FACP

Topics: Palliative Care Delivery/Operations: Successful development of pharmacy roles on a palliative care team

\section{Description:}

The core team members of a palliative medicine team include physicians, nurse practitioners, nurses, social workers, and chaplains. Although pharmacists have been identified as valuable team members, most inpatient palliative teams have not been able to have a full-time pharmacist in a small community hospital setting. Our program illustrates a collaborative agreement whereby a palliative trained pharmacist is integrated into the palliative medicine service. The pharmacy department provides 24-hour inpatient coverage and has a pharmacy managed pain protocol that is used to ensure patients have access to pharmacists all the time. A palliative trained pharmacist works with the palliative medicine team Monday through Friday, $8 \mathrm{am}$ to $5 \mathrm{pm}$. The pharmacist participates in the interdisciplinary team meeting three times weekly with the palliative medicine team, follows assigned patients, and focuses on patients with symptom management, endof-life care, and any medication education needs. The palliative pharmacist signs off to the $\mathrm{pm} / \mathrm{night} /$ weekend pharmacist for necessary follow-up. The pharmacists document in the electronic medical records. A palliative team member is always on call; however, with the pharmacist pain protocol being available 24/7, it complements the team and has proven to be extremely effective and rewarding, enhancing patient care. 
27. Formation of the Global Palliative Care Quality Alliance: A Cooperative Group Dedicated to the Advancement of Quality within Palliative Care

Duke Cancer Institute

Durham, NC

Team/Contact Information:

Arif Kamal, $M D$

Director, Quality and Outcomes

Duke Cancer Institute

210 Graduate Court

Durham, NC 27713

(919) 681-0333

Arif.kamal@duke.edu

Project Team Members:

Jon Nicolla, MBA

Fred Friedman

Laura Roe

Topics: Coalition and Advocacy: Regional and National Palliative Care Initiatives (e.g., statewide coalitions/political advocacy)

Description:

The Global Palliative Care Quality Alliance is a volunteer network of community-based palliative care organizations striving to enhance the quality of palliative care delivery through coordinated data collection, regular sharing of best practices, and collaborative quality improvement. The alliance prioritizes quality measurement and sustainable improvements in community-based palliative care settings, through a unique partnership between community-based practices and academic medical centers. Participating sites collect data on quality near point of care using an alliance-derived software system; those data are then used to inform quality measure adherence, benchmarking across sites, performance improvement efforts, and health services and outcomes research. To date, the alliance has collected data on quality on over 34,000 patient encounters and has completed three impactful rapid cycling quality improvement initiatives. Further, research on disparities among racial populations, differences in unmet needs by disease types (e.g., cancer versus non-cancer), and care locations (inpatient versus outpatient versus home), and development of novel caregiver quality measures have significantly impacted how participating sites provide palliative care. Additionally, regular conference calls, a national meeting on quality, and collaborative participation in projects ensure engagement and a sense of community by all.

28. The Challenges of End-of-Life Care for Homeless Veterans

Eastern Colorado VA Health Care System

Denver, CO

Team/Contact Information:

Evelyn Hutt, MD

Eastern Colorado VA Health Care System

1055 Clermont Street

Denver, CO 80220

(720) 857-5112

evelyn.hutt@va.gov

Project Team Members:

Jacqueline Jones, $R N, P h D$

Mary Weber, RN, PhD

Karen Albright, PhD

Cari Levy, MD, PhD

Thomas O'Toole, MD

Emily Whitfield, PhD

Sung-Joon Min, PhD

Hannah Dischinger, BS
Topics: Palliative Care Delivery/Operations: Innovative and emerging community-based palliative care models (e.g., home/ office/clinic/long-term care)

Description:

Homeless veterans approaching the end of life (EOL) may not be getting the care they need. This study surveyed existing Department of Veterans Affairs (VA) program providers for an overview of how homeless veterans at EOL are currently getting care. Focus groups and key informant interviews then were conducted with veterans, multi-disciplinary providers, and community and VA leadership at four geographically dispersed sites. A National Program and Policy Development Forum was convened to address the challenges uncovered.

Main Findings: VA Medical Centers treated an average of 6.5 homeless veterans at EOL annually. Interviewees and focus group participants emphasized: (1) in spite of homeless veterans' declining health, current housing options are too often limited to places that insist on functional independence, sobriety, and non-use of pain medication; (2) pain management in the context of unstable housing, behavioral health issues, and addiction is challenging; (3) discontinuity of care between systems restricts EOL care; (4) VA regulations pose significant challenges to collaboration with community providers; and (5) dedicated homeless and EOL program staff collaborate informally to provide care.

The Forum is developing policy recommendations, education, and pilot programs to maximize VA-community partnerships and employ peer support by formerly homeless veterans to address these challenges.

\section{CARES-Palliative Care Program in the Nursing} Home

Eastern Virginia Medical School/Sentara

Norfolk, VA

Team/Contact Information:

Deborah A. Morris, MD

Assistant Professor

Eastern Virginia Medical School/Sentara

825 Fairfax Avenue 201

Norfolk, VA 23322

(757) 446-7040

damorri3@sentara.com

Project Team Members:

Lauren Mazzurco, DO

Marissa Galicia-Castillo, MD

Topics: Palliative Care Delivery/Operations: Innovative and emerging community-based palliative care models (e.g., home/ office/clinic/long-term care)

\section{Description:}

Caring About Residents' Experiences and Symptoms (CARES) is a collaborative approach to providing palliative care in the nursing home. Sentara Life Care and Eastern Virginia Medical School partnered together to develop and sustain a collaborative palliative care program for nursing home residents at 197 bed nursing facility. The CARES team consists of EVMS palliative medicine physicians and a Sentara chaplain who partner with Sentara Life Care physicians, nurses, social workers, and staff to provide comprehensive care for residents facing life-limiting medical illness. With this collaborative program, residents facing life-limiting illness are better able to understand their condition and choices for care, while they receive intensive management of their symptoms and additional social and spiritual support for themselves and their families. We present facility-centered outcomes and resident-centered outcomes including hospice referral, mortality, location of death, hospitalizations, and change in care 
plan to address symptom needs. The CARES program immediately and directly impacts the care available and received by nursing home residents. As no standardized approach to providing palliative care to nursing home residents has been adopted in community nursing homes, we hope our experience can be used to assist others in meeting this need in their own communities.

\section{Mandatory versus Optional Fields for PC Quality Metrics in EMRs}

Emory Palliative Care Center, Emory University

Atlanta, GA

Team/Contact Information:

Zachary Binney

Emory Palliative Care Center, Emory University

1518 Clifton Road

Atlanta, GA 30322

(404) 803-6877

zbinney@emory.edu

Topics: Data and Outcomes: Successful use of metrics in community or hospital settings

\section{Description:}

To improve documentation of quality metrics a multidisciplinary team of clinicians designed new documentation templates in our electronic medical record (EMR) to capture key palliative care (PC) quality metrics. We sought to determine what proportion of initial inpatient PC consults included quality metric data when the note fields were mandatory versus optional in three Southeastern academic hospitals.

We compared documentation percentages from January to April (mandatory fields) with June to July 2015 (optional fields). There were 1428 initial consults and 2636 follow-up visits during the "mandatory period" and 576 and 1340 during the "optional period." Documentation rates were $100 \%$ when mandatory. During the optional period, the documentation rates were: dyspnea screen, $82 \%$; nausea screen, $73 \%$; offers of spiritual support, $80 \%$; and surrogate name and phone documentation $89 \%$ and $80 \%$, respectively. Opioid use was documented in $59 \%$ of initial consults when optional; clinicians reported positive opioid use in $45 \%$ of consults when mandatory but only 29\% when optional. Dyspnea and nausea were "unable to be assessed" in $\sim 30 \%$ of mandatory versus $\sim 20 \%$ of optional consults. Of mandatory phone numbers $5 \%$ were invalid entries.

Although the notes were designed with multidisciplinary clinician input to optimize workflow, some objected to the mandatory fields. Making these optional reduced documentation by 11 to $27 \%$.

31. The Comfort Care Home and Mercy Doula Training Program: Giving Individuals the Ability to Die with Dignity Erie County Medical Center

Buffalo, NY

Team/Contact Information:

Kathleen T. Grimm, MD

Consultant

Erie County Medical Center

462 Grider Street

Buffalo, NY 14215

(716) 472-0035

kgrimm1@ecmc.edu

Project Team Members:

Elder Manuel Wiggins

Rev. Garney Davis Jr.

Chloe Emerling

Sandra Lauer
Topics: Palliative Care Delivery/Operations: Innovative and emerging community-based palliative care models (e.g., home/ office/clinic/long-term care)

\section{Description:}

Palliative care embraces the principle that care for the dying must be equitable and accessible to all, and will deliver care based on the expressed value of most Americans-to die at home. The ability to be in a home where palliative care can take place is lacking for many marginalized and disenfranchised patients. These patients include the mentally ill, the homeless, frail elderly living alone, and others with limited social structure. When these patients are eligible for hospice care, the barrier to receiving this care is that they lack the social structure for caring in their own home, or in a home in their community. Erie County Medical Center (ECMC) sees many of these patients, as a safety net hospital that is also a behavioral health center. ECMC is creating an alternative to institutional care that can fill this large gap for our patients. This gap is being filled by the creation of a comfort home for the dying - a community based home, with care given by volunteers from the community. This community home will care for two to three patients at a time as they die, in a home that is supported with volunteers, many trained as mercy doulas.

\section{Value-Based Conversations in Diverse Communities \\ Erie Country Medical Center}

Buffalo, NY 14221

Team/Contact Information:

Kathleen T. Grimm, MD

Consultant

Erie County Medical Center

462 Grider Street

Buffalo, NY 14215

(716) 472-0035

kgrimm1@ecmc.edu

Project Team Members:

Elder Manuel Wiggins

Rev. Garney Davis Jr.

Chloe Emerling

Sandra Lauer

Topics: Communication: Communication best practices (e.g., teaching goals of care conversations/advance care planning/ family meetings)

\section{Description:}

There is well-documented disparity in health care outcomes in minority groups, and this disparity extends to end of life. The Conversation Project at Erie County Medical Center (ECMC) is a palliative care outreach team working on bridging the continuum of conversation around end-of-life care that is taking place in and around urban Buffalo, NY. This outreach team includes members of the community that reflect the diverse population that surrounds the medical center. Surveys that have measured the effectiveness of this team have challenged thinking re: the established belief that communities are not willing to engage in these conversations. Rather, outreach results show that the community not only wants to hear about this conversation, but is showing a readiness to action that needs to be translated into documentation by the health care community. The inclusion of these community members, as part of the palliative education team, increases community engagement that is respectful, culturally sensitive, and avoids stereotyping. These non-clinical team members work as "connectors"connecting individuals to care in a manner that is respectful of each person, understanding the culture, beliefs, and values that 
shape what matters most to each individual, and creating measurable change.

33. Extending Your Reach-Using Video Visits at Home Fairview Home Care and Hospice

Minneapolis, MN

Team/Contact Information:

Jody Ramer Chrastek, DNP

Fairview Home Care and Hospicel

University of Minnesota Masonic Children's Hospital

2450 26th Avenue South

Minneapolis, MN 55406

(612) 728-2389

jchrast1@fairview.org

Topic: Palliative Care Delivery/Operations: Innovative and emerging community-based palliative care models (e.g., home/ office/clinic/long-term care)

\section{Description:}

The Internet and immediate video connections are commonplace in today's society, but not so in home care and hospice services. Video visits are a valuable resource for communication, education, and support. (Oliver, D. et al. 2012). Over $80 \%$ of Minnesota's urban population has Internet access and a computer (www.ruralmn.org, 2012). Video visits allow for non-verbal communication to take place quickly and effectively providing a way to intervene when weather, traffic, or staffing issues cause delays. This pilot program explores the effects of providing real-time video connection between staff lap top and patients' or families' home computer. The video platform is a HIPPA-secure website, provides a virtual "office" waiting room, and requires no down load, allowing family members, the medical team, and others to meet virtually at any time. Data were collected from both staff and patients/families on the effect of video visits on anxiety level, feelings of connectedness to the team, and confidence in care. This project shows how integrating video visits into a larger plan of care can benefit patients, families, and the program. Time line, strategies, and lessons learned will be discussed.

34. Identifying and Addressing Nursing Barriers in EOL Care

Greenwich Hospital

Greenwich, CT

Team/Contact Information:

Donna E. Coletti, MD

Medical Director

Greenwich Hospital

5 Peryridge Road

Greenwich, CT 06830

(203) 531-0395

donna.coletti@greenwichhospital.org

Project Team Members:

M. Hansley, APRN

B. Leafe, MSN, RN

K. Acevedo, $R N$

K. Culmone, MSN, RN

H. Archer, MD, PhD

Topics: Education: Targeting Education: Pain management and communication skills

Description:

By identifying common barriers to care at our community hospital then training palliative care resource nurses (PCRNs) in end-of-life (EOL) management, an educational program for medical and oncology nurses was developed and implemented by the palliative care team. The initiative was prompted by the PC team noting disparities in their patients' care directly related to the nurse's knowledge base and personal comfort level in administering as needed (prn) narcotic and anxiolytic medications at EOL.

The PC team, nursing administration, and nursing management identified and trained a group of medical and oncology nurses as PCRNs to act as a primary resource to their peers in patient assessment, active symptom management, and cultural differences. In order to determine effectiveness and comfort level with this subject, a needs assessment tool developed by our team that was previously used in a hospital-wide PC survey was administered that involved answering specific PC-related topics with a follow-up assessment 6 months after the initial one. The PC team provided education on patient assessment, symptom management, pharmacology, role modeling, and implementation of the CARES model, first developed at the City of Hope. The program includes quality measures and will be included in the hospital documents in applying for Magnet certification.

\section{Integration and Enculturation of a Palliative Care Program}

Inova Fair Oaks Hospital

Fairfax, VA

Team/Contact Information:

Lynne Kennedy, PhD

Inova Fair Oaks Hospital

Service Lines

3600 Joseph Siewick Drive

Fairfax, VA 22033

(703) 391-4841

lynne.kennedy@inova.org

Project Team Members:

Thomas Sullivan MD

Topics: Data and Outcomes: Data demonstrating depth of palliative care integration in ACO/PCMH/bundling and other risk-bearing health care delivery frameworks

Description:

Hospital, Medicine, Chaplaincy, Social Work

Background: Inova Fair Oaks Hospital is a 182-bed community hospital located in Fairfax, VA. In 2009 one provider began seeing patients using a palliative care consult model, which led the intensivists in our ICU to request palliative care participation at ICU inter-disciplinary morning rounds. The satisfaction of the medical team in ICU resulted in discussions with hospital senior leadership to sponsor a palliative care team, charged with the goals of improving the quality of patient care and of achieving Disease Specific Certification for Advanced Palliative Care from the Joint Commission. Certification was achieved in May 2012 and re-certification in May 2015.

The result of this systematic approach to platform development led to: Consistent growth in palliative care referrals, incorporation of palliative care as a "standard of care," positive impact on hospital patient satisfaction surveys, improved pain control, improved nursing satisfaction with interdisciplinary team support and cooperation, and shorter length of stay with reduced 30-day re-admissions.

36. RPM and Palliative Care Improves Patient Outcomes Intermountain Homecare and Hospice

South Jordan, UT

Team/Contact Information:

Kimberly A. Lane, MSN

Hospice Nurse Administrator 
Intermountain Homecare and Hospice

11520 S. Redwood Road

South Jordan, UT 84095

(385) 887-6782

Kim.Lane@imail.org

Project Team Members:

E.L. Shadbolt

K.A. Lane

A.J. Gilbert

L.A. Musgrave

Topics: Data and Outcomes: Using technology to improve efficiency and outcomes

Description:

$\mathrm{KR}$ is a 57-year-old patient with high emergency room (ER) and hospital utilization preceding her admission to homecare. KR was started on a remote patient monitoring (RPM) program, with a home health case manager. With RPM KR's vital signs were monitored three times daily by a nurse, and KR was able to see her vital sign trend with relation to her diet and activity. One month post-implementation her ER and hospital utilization had decreased, but she continued requiring twice daily nursing visits. KR then began the home-based palliative care program. The palliative care case manager was able to work with KR to identify her goals and understand her barriers to compliance. The case manager was able to personalize and target the education KR received. With this partnership, KR understood the education and her compliance with medications and diet improved. Within one month the nursing visits were decreased to daily with one hospitalization due to acute renal failure, and no ER visits. Within 3 months nursing visits were decreased to three times weekly with one hospitalization due to acute myocardial infarction.

37. Integration into a County/Safety-Net Teaching Hospital John Peter Smith Health Network

Fort Worth, TX

Team/Contact Information:

S. DiAnn Young, NP

John Peter Smith Health Network

1500 S. Main Street

Fort Worth, TX 76179

(817) 702-6825

SYoung01@jpshealth.org

Project Team Members:

Matthew L Rios, MD

DiAnn Young, MSN, APN-BC

Topics: Palliative Care Delivery/Operations: Integrating palliative care services (e.g., across a health-system/patient-centered medical home/assisted living/nursing homes)

Description:

The palliative care inpatient consultation program is a Delivery System Reform Incentive Payment (DSRIP) Project under the Texas 1115 Healthcare Transformation Waiver. Prior to this initiative the hospital had no organized palliative care program. This session will provide an overview of the pre-work, implementation and integration strategies, identification of the challenges, ongoing solutions, opportunities, lessons learned, and positioning your program for growth.

\author{
38. Making the Case: Data Collection Made Easy \\ Lehigh Valley Health Network \\ Allentown, PA \\ Team/Contact Information: \\ Jennifer Allen \\ Palliative Medicine Consulting Physician
}

Lehigh Valley Health Network

2166 South 12th Street, Suite 402

Allentown, PA 18103

(610) 402-8106

jennifer_e.allen@lvhn.org

Project Team Members:

D. Ray

D. Stevens

R. Schira

Topics: Data and Outcomes: Successful use of metrics in community or hospital settings

\section{Description:}

Background: Palliative care consultation improves quality and decreases hospital costs. Demonstrating the contribution of palliative care to stakeholders and administrators is challenging.

Methods: Based on published metrics, we developed a webbased tracking tool to improve adherence to standard consult components and quality interventions. It was accessible through a link in our electronic medical record. Providers prospectively completed the tool for each consult. Metrics were analyzed via Excel spreadsheet.

Results: Between July 2014 and June 2015, 2949 consults were performed with 2286 having completed tools $(77.5 \%$ completion rate). Although $<10 \%$ of consults requested were for symptom management, uncontrolled symptoms were identified and treated in $26 \%$ of cases. Eliciting patient/family values resulted in less aggressive medical care at end of life. Fortyeight percent of patient/families chose "no CPR" status; $23 \%$ of patients seen were referred to hospice services; $10 \%$ were transferred out of a critical care setting; and in $18 \%$ there was a decrease in resource utilization.

Conclusion: Use of the tool was efficient to quantify interventions commonly performed in palliative care consultation.

Implications: The data tracking tool may be adapted to specific stakeholder interests, whether it is cost based or quality based.

39. Palliative Care Integration into Medical Education Loma Linda University Health

Loma Linda, CA

Team/Contact Information:

Michelle Rhiner, DNP

Nurse Practitioner Center for Palliative Care

Loma Linda University Health

11175 Campus Street

Loma Linda, CA 92350

(909) 558-7196

mrhiner@llu.edu

Project Team Members:

Neal E. Slatkin, $M D$

Gina Jervey Mohr, MD

Topics: Education: Targeting Education: Pain management and communication skills

\section{Description:}

Objective: To explore differences of self-perceived competencies, ethical concerns, knowledge, and attitudes regarding palliative care between medical students who took a palliative care rotation and those who did not.

Methods: A mixed methods, non-experimental study utilizing fourth-year medical students completing tests in knowledge, self-perceived competence, and ethical concerns, and attitudinal questions regarding palliative care.

Results: Forty-three medical students participated. Those who completed the palliative rotation had significantly $(\mathrm{t}=41=3.56$, 
$p<0.001)$ higher scores in overall knowledge. Factor analysis of the 26-items yielded six factors with statistical differences being observed in four factors. For ethical concerns, the significant difference was noted in the area of withdrawing artificial hydration. Students who completed the rotation reported a much broader understanding and application of palliative care concepts. Students who did not take the rotation had a narrow view of the benefits and application of palliative care, seeing application only in end-of-life situations.

Conclusions: The information about palliative care is important during medical school. Mentoring by the palliative care team was seen as valuable, therefore didactic information should be coupled with a formal palliative care rotation to best meet the needs of the seriously ill individual and family.

\section{Let's Talk: A Fun Way to Teach Physician Commu- nication \\ Long Beach Memorial Medical Center \\ Long Beach, CA}

Team/Contact Information:

Katy Hyman, MDIV

Program Coordinator, Palliative Care

Long Beach Memorial Medical Center

2801 Atlantic Avenue

Long Beach, CA 90806

khyman@memorialcare.org

Project Team Members:

Casey Hudson, LCSW

Denis McCourt, MFA

Sheryl Faulk, BCC

Rebecca Yamarik, MD

Wendy Linderholm, PsyD

Shannon Moore, MD

Topics: Education: Targeting Education: Pain management and communication skills

\section{Description:}

Training attending and resident physicians in the art of family conferences and patient/family communication is a vital component of palliative care outreach. Our team partnered with local professional actors to bring live training to the clinicians. Thorough character studies provided the actors with background information and scripting; basic case studies (with medical, familial, social histories) gave the physicians and interdisciplinary team enough information to begin the conversation. In order to make the learning accessible, the session was designed to take only one hour from each clinician's day. The room was set up with a basic video recorder, a few chairs in a circle for the meeting, and other chairs set back for the observers (PC physicians and others trained in debriefing). The physician and other team members arrived and were given 5 minutes to review the case study and have a brief huddle to plan their meeting, then the actors came in and the meeting proceeded. After 20 to 30 minutes, we debriefed the meeting, inviting reflection and feedback from the participants and observers. Video recordings were made available through a private YouTube channel for later viewing and reflection by the participants.

41. The Integration of Palliative Care in Rural Tennessee Maury Regional Medical Center

Columbia, TN

Team/Contact Information:

Tammy Stokes, BSN

Palliative Care Nurse Navigator

Maury Regional Medical Center

3 S. Park Grove Road
Columbia, TN 38464

tstokes@mauryregional.com

Topics: Palliative Care Delivery/Operations: Integrating palliative care services (e.g. across a health-system/patient-centered medical home/assisted living/nursing homes)

\section{Description:}

Maury Regional Medical Center (MRMC) is located in Columbia, TN, and is licensed for 255 acute care beds. MRMC is the largest hospital between Nashville, TN, and Huntsville, AL. MRMC serves greater than six counties in Tennessee with a population of more than 200,000 people living in rural settings. When senior leadership agreed to start a palliative care program at MRMC it became clear that the success of the program would only come through building relationships within communities served to properly access palliative care needs and to discover innovative ways to deliver care in rural middle Tennessee. A palliative care network was developed to lead the charge in integrating palliative care services. This network was comprised of health care leaders from local hospice organizations, nursing homes, and surrounding hospitals and their focus was to develop integrative ways to deliver palliative care across the health care continuum from the hospital to the patient's home, assisted living, or nursing homes. Members of the network were responsible for assessing the needs within the community where they live and work. The information gathered was vital in developing a system to deliver palliative care services to patients living with chronic illness in rural middle Tennessee.

\section{Using the "Surprise Question" in Primary Care Mayo Clinic, Office of Population Health Management Rochester, MN \\ Team/Contact Information: \\ Karina M. Squire, $R N$ \\ Staff Nurse \\ Mayo Clinic, Office of Population Health Management 1025 2nd Avenue}

Rochester, MN 55901

(541) 663-6741

Squire.Karina@mayo.edu

Project Team Memebrs:

Elizabeth L. Bechtum, MNHP, RN

Erin Henderson, MHA, RN

Kate J. Larson, MAN, RN

Jennifer Schreiber, MA

Mary Thelen, MSN, RN, CHPN

Cory Ingram, MD, MS FAAHPM

Topics: Palliative Care Delivery/Operations: Innovative and emerging community-based palliative care models (e.g., home/ office/clinic/long-term care)

\section{Description:}

A new model of health care delivery has been proposed that incorporates the core principles of palliative care into the primary care setting. A question has been identified that helps recognize individuals who would benefit from palliative care measures. This question - the surprise question (SQ) — asks, "Would you be surprised if the patient died within the next year?" If the answer is no, the provider is triggered to initiate palliative measures with the patient. These measures include the following: advance care planning, spiritual assessments, symptom assessment and management, and a routine Patient Health Questionnaire (PHQ)-2 depression screen. Because this is a relatively new innovation, research specific to use of the SQ in the primary care setting is limited but promising. A quality improvement project is underway to assess use of the SQ in primary care as a 
trigger for palliative care measures. During a daily multidisciplinary huddle, participants will be asked to consider the SQ and identify patients who would benefit from palliative care. The use of a team-based model in primary care assures that Mayo Clinic will be well positioned to deliver relationship-based palliative care.

43. Playing Well in the Sandbox: Growing an Outpatient Palliative Care Clinic While Enhancing Collaboration with Interventional Pain Clinicians

Mayo Clinic

Rochester, MN

Team/Contact Information:

Jacob J. Strand, MD

Director, Outpatient Palliative Care

Mayo Clinic

200 First Street SW

Rochester, MN 55902

(507) 294-1094

strand.jacob@mayo.edu

Project Team Members:

Kelly $W u, M D$

Kelly J. Christensen, PA-C

Topics: Methods of training in primary/generalist palliative care (e.g., palliative care training for hospitalists and unit nurses in communication/symptom management) (PCP)

\section{Description:}

Pain is one of the most commonly experienced and feared symptoms in patients facing a serious illness and a common reason for referral to a palliative care service. Palliative care teams are able to control symptoms of pain with pharmacologic and nonpharmacologic adjuvant therapies in most circumstances. However, a substantial minority of patients will have inadequate analgesia and/or intolerable side effects with systemic therapies (up to $25 \%$ in patients with cancer pain). For these patients, minimally invasive interventional pain therapies can be significantly beneficial. However, despite these benefits, a number of studies demonstrate a lack of collaboration between palliative care and interventional pain providers, resulting in fewer patients receiving potentially beneficial interventions. Lack of mutual understanding about one another's role and potential benefits and concern about losing patients to another's practice have been postulated as potential reasons for this lack of collaboration.

The outpatient palliative care clinic (named SYMPAQ: Symptom Management, Pain \& Quality of Life) at Mayo Clinic in Rochester, MN, was established in 2008 and had 89 patient visits in its first year. By 2009, the number of patient visits more than doubled to 219. Patient visits doubled again by 2011 to 512 and increased to 681 visits by 2013 reflecting an average yearly increase in patient visits of $60 \%$ per year since its inception. During this growth, practice leaders in both groups have purposefully planned ways for the two groups to collaborate, learn from one another, and in turn avoid potential competitive pitfalls. Joint teaching conferences, pre-scheduled visits with providers from both specialties, and co-rounding models have helped enhance collaboration. Early data also suggest that although consultations to the palliative care practice have increased, so too have the number of procedures referred to our interventional pain clinicians.

\author{
44. Are Families Satisfied after Receiving "Bad News"? \\ MedStar Health \\ Columbia, MD \\ Team/Contact Information: \\ Kathryn A. Walker, PHARMD, BCPS, CPE
}

MedStar Health

Clinical Director of Palliative Care

201 East University Parkway

33rd Street Building, Suite 415

Baltimore, MD 21218

kathryn.walker@medstar.net

Project Team Members:

Joshua Fabie, MS

Christopher Kearney, MD

Topics: Communication: Communication best practices (e.g., teaching goals of care conversations/advance care planning/ family meetings)

Description:

This study was designed to describe the impact of PC family meetings on family satisfaction in our inner-city community teaching hospital.

Methods: Families of patients participating in initial family meetings with the PC team between June 23 and July 24, 2015 were offered the opportunity to complete a survey on an electronic tablet following the meeting. Questions included a Likert scale to report satisfaction with communication before and after the meeting. Clinicians in the meeting were also surveyed to record topics discussed in the meeting. Data were analyzed using descriptive statistics. A net promoter score (NPS, range -100 to +100 ) was calculated.

Results: Twenty-eight families ( $n=59$ respondents) participated in this study. Most were the patient's primary spokesperson $(58 \%)$. The most common topics discussed in meetings were goals of care (96\%), end-stage/terminal prognosis $(79 \%)$, and pain/symptom management $(63 \%)$. The greatest differences in NPS were in the following areas: met needs $(\Delta 63)$, trust $(\Delta 53)$, and courtesy and respect $(\Delta 50)$. The overall NPS of all six elements of satisfaction in the survey was 37 before and 81 following the PC family meeting.

Conclusion: Despite discussing serious topics in family meetings with the palliative team, families reported high levels of satisfaction compared with prior communication.

\section{The Impact of Palliative Care Decisions at Hospital Discharge on RA \\ MedStar Health \\ Columbia, MD}

Team/Contact Information:

Kathryn A. Walker, PHARMD, BCPS, CPE

MedStar Health

Clinical Director of Palliative Care

201 East University Parkway

33rd Street Building, Suite 415

Baltimore, MD 21218

kathryn.walker@medstar.net

Project Team Members:

Joshua Fabie, MS

Christopher Kearney, MD

Topics: Data and Outcomes: Successful use of metrics in community or hospital settings

\section{Description:}

This study describes the impact of early palliative care (PC) hospital consultation and the discharge plan of care on the rate of readmission (RA).

Objectives: 1) compare RA rates and charges for patients choosing a PC plan versus non-palliative after discharge; 2) compare differences in hospital charges between early consultations and plans of care. 
Methods: We included all patients receiving PC consultation and surviving to discharge in three hospitals (12/12-4/13). Palliative care plan of care (PCPOC) was defined as postdischarge plans that focused primarily on comfort (e.g., hospice) and non-palliative plans of care (NPPOC) represented usual care. Early consultations were performed $\leq 3$ days on index hospitalization.

Results: One hundred eighty patients were included in this study and 53\% were consulted early. Overall, 64\% agreed to a PCPOC at discharge. Hospital charges were less for early consultations $(\triangle \$ 22,566)$ and for those with a PCPOC. Thirtyday mortality was higher in the PCPOC group ( $80 \%$ versus $29 \%$ ) and overall RA rates were higher in the NPPOC group ( $42 \%$ versus $7 \%$ ). When adjusted for mortality, RA rates remained higher in the NPPOC group (45\% versus $21 \%$ ). Thirtyday RA charges were higher per patient $(\Delta \$ 7,031)$ and per RA $(\Delta \$ 9,646)$ for the NPPOC group.

\section{Palliative Care Consultations for Orthotopic Heart Transplant Recipients}

MedStar Heart and Vascular Institute

MedStar Washington Hospital Center

Washington, DC

Team/Contact Information:

Hunter Groninger, MD

Director, Palliative Care

MedStar Washington Hospital Center

Department of Medicine

Section of Palliative Care

110 Irving Street $N W$

Washington, DC 20010

(703) 343-5859

hunter.groninger@medstar.net

Project Team Members:

M. Aggarwal, $M D, M P H$

K. Walker, PharmD

G. Ruiz, $M D$

J.T. Panke, NP, ACHPN

R.M. Holder, PharmD

A. Kelemen, LICSW

Topics: Palliative Care Delivery/Operations: Approaches to palliative care delivery for specific population types (e.g., heart failure/cancer/dementia/frailty)

\section{Description:}

Cardiac transplantation is associated with significant morbidity, mortality, and supportive care needs. Nevertheless, partnerships between heart transplant and palliative care (PC) teams are extremely rare; data remain limited on how such services benefit cardiac transplant patients and families. To describe indications for PC consultation and PC interventions in cardiac transplant recipients, we retrospectively reviewed 10 cardiac transplant recipients who received PC consultation between January and July 2015. Reason for consultation, pain and symptom assessment (on day 0,2), estimated prognosis, PC interventions, and disposition were collected. Sixteen PC consults took place for 10 cardiac transplant recipients. The primary reason for consultation was symptom management (pain $86 \%$, non-pain symptoms $14 \%$ ). Fifty-seven percent of patients had moderate-severe symptoms at presentation. Symptom scores decreased to none-mild within 48 hours of initial consultation in half of these cases. Additional PC interventions included psychological counseling (19\%), spiritual care (13\%), disease state education (6\%), and medical proxy determination $(6 \%)$. Most hospitalized patients were discharged; however, two required end-of-life care during hospitalizations. In this series, cardiac transplant recipients experienced notable symptom burden, responded well to interventions to treat symptoms, and required additional PC interventions. Future research should further describe PC consultation's role and benefit in cardiac transplantation.

47. Palliative Care Integration for Patients Receiving Left Ventricular Assist Device as Destination Therapy

MedStar Heart and Vascular Institute

MedStar Washington Hospital Center

Washington, DC

Team/Contact Information:

Hunter Groninger, MD

Director, Palliative Care

MedStar Washington Hospital Center

Department of Medicine

Section of Palliative Care

110 Irving Street NW

Washington, DC 20010

(703) 343-5859

hunter.groninger@medstar.net

Project Team Members:

M. Aggarwal, $M D, M P H$

K. Walker, PharmD

G. Ruiz, $M D$

J.T. Panke, NP, ACHPN

R.M. Holder, PharmD

A. Kelemen, LICSW

Topics: Palliative Care Delivery/Operations: Approaches to palliative care delivery for specific population types (e.g., heart failure/cancer/dementia/frailty)

Description:

Left ventricular assist device as destination therapy (LVADDT) can be life-prolonging therapy available to select patients with advanced heart failure; it is also associated with significant morbidity, mortality, and supportive care needs. MedStar Washington Hospital Center began integrating palliative care services for LVAD-DT patients in January 2015. Using the electronic health record, we retrospectively reviewed all LVAD-DT patients who received PC consultation between January and July 15, 2015. Data collected included reason for PC consultation, pain and symptom assessment (day 0, day 2), PC interventions, and disposition. Thirty-one LVAD-DT patients received $\mathrm{PC}$ consultations. The primary reason for $\mathrm{PC}$ consultation was pain (25/31); median time to consultation was 9 days (median total length of stay was 26 days). Eighty-one percent of patients had moderate-severe symptoms at time of consult, which decreased to $20 \%$ by day 2 . Additional PC interventions included psychological (19\%), spiritual (32\%), and/ or medication (87\%) counseling as well as disease state education $(26 \%)$, proxy determination $(13 \%)$, and change in code status (3\%). Most patients went home $(58 \%)$ or to rehab (39\%) on discharge; $3 \%$ died in the hospital. We conclude that LVADDT candidates experienced notable symptom burden and required multiple PC interventions, highlighting the value of PC integration.

\author{
48. Regional Survey of Advanced Heart Failure Care in \\ Hospice \\ MedStar Washington Hospital Center \\ Washington, DC \\ Team/Contact Information: \\ Renee M. Holder, PHARMD
}


Clinical Pharmacist

MedStar Washington Hospital Center

110 Irving Street $\mathrm{NW}$

Washington, DC 20010

(202) 877-7841

renee.m.holder@medstar.net

Project Team Members:

K. Walker, PharmD

G. Ruiz, $M D$

J.T. Panke, NP, ACHPN

A.M. Kelemen, LICSW

$H$. Groninger, $M D$

Topics: Palliative Care Delivery/Operations: Approaches to palliative care delivery for specific population types (e.g., heart failure/cancer/dementia/frailty)

Description:

Background: Advanced heart failure (AHF) therapies, in cluding inotropes and ventricular assist devices (VADs), may improve symptoms, function, and longevity for select patients. The role of these therapies in hospice care is not well defined.

Research Objective: To describe the variation in practice as a needs assessment in improving care for AHF patients.

Methods: At an AHF summit, an 18-item multiple-choice survey was distributed to metropolitan DC area hospice leaders. Survey topics included demographic information about the hospice, experience caring for AHF patients, and opinions on barriers and preferences for training.

Results: Nineteen hospice leaders were represented and completed the survey (100\% response rate). Respondents were in administrative (11/19) and clinical (11/19) leadership roles. Most respondents felt access to hospice for AHF patients was adequate/somewhat adequate (13/19), but timing of referrals was late (18/19). Fifteen (79\%) reported not having algorithms for AHF management. Ten (53\%) reported inotropes were cost prohibitive. Of those, $90 \%$ would cover inotrope costs if comparable to the cost of parenteral opioids. Fourteen $(74 \%)$ reported lack of experience with inotropes, and $18(95 \%)$ indicated interest in training.

Conclusion: We identified lack of experience, cost, and late referrals as barriers to providing advanced therapies for AHF in hospice settings.

\section{Impact of Supp. Med. on Symptom Management in IP Setting}

Memorial Hermann Physician Network and Symptom Management Consultants

Houston, TX

Team/Contact Information:

Kathryn Lowe, MHA

Administrative Manager

Memorial Hermann Physician Network and Symptom Management Consultants

909 Frostwood Suite 1.408

Houston, TX 77024

(336) 918-9499

kathryn.lowe@memorialhermann.org

Topics: Other

Description:

Symptom data from eight categories were analyzed for comparison of the severity of symptoms, from the time Supportive Medicine (SM) was consulted, until time of patient discharge. Symptom data from a patient population of 345 was used for analysis. Patients who did not present with symptoms or whose length of stay was less than 2 days post-SM consult were not included. Symptoms were tracked using a 0 to 3 scale based on severity of symptoms, with " 0 " representing no symptoms present, and a " 3 " indicating severe symptoms. If patients were unable to describe their symptoms, the ratings were based on the observations of the nurse coordinator. Data were tracked over a 16-month period at Memorial Hermann Southeast Hospital in Houston, TX. The sample sizes varied based on the number of patients who presented with a particular symptom. Data anlysis displayed a statistically significant improvement in symptoms in each of the eight measured categories at a confidence interval of $p<0.05$. The largest improvement was seen in nausea, which decreased from a rating 1.11 on the initial date of consult to 0.23 upon discharge $(n=95)$. The mean improvement across all symptoms was 0.70 points between date of consult and discharge.

50. A System Approach to Inpatient Palliative Care Services

Ministry Health Care

Appleton, WI

Team/Contact Information:

Olumuyiwa O. Adeboye, MD

System Medical Director

Ministry Health Care

222 W. College Avenue

Appleton, WI 54911

(920) 628-9217

Olumuyiwa.Adeboye@ministryhealth.org

Project Team Members:

Ann K. Patek, RN, MSN

Topics: Palliative Care Delivery/Operations: Innovative hospital-based palliative care models

\section{Description:}

Ministry Health Care has recognized the need to expand the reach of palliative care services and identified these services as a strategic imperative. Palliative care aligns well with the values and mission of our Catholic system and provides an important component of care as we move to align our work with a population-based health model of service delivery.

To facilitate this work the system implemented a dyad leadership team and charged it with implementing a multidisciplinary inpatient palliative care consult team in 7 hospitals located in the central, eastern, and northern parts of Wisconsin. This poster describes the work to implement a standardized palliative care service that is deployed locally. Challenges of multiple EHR applications, geographic distance, and lack of understanding/acceptance of palliative care are considered in the narrative.

51. Advance Care Planning in a Tertiary Care Cancer Center

Moffitt Cancer Center

Tampa Florida, FL

Team/Contact Information:

Sarah Thirlwell, MS, MSN, RN

Moffitt Cancer Center

12902 Magnolia

MRC-SCM

Tampa Florida, FL 33612

(813) 745-6045

sarah.thirlwell@moffitt.org

Project Team Members:

Nancy Newman, LCSW

Diane Portman, MD, FAAHPM 
Topics: Palliative Care Delivery/Operations: Integration of palliative care services in cancer centers/dialysis centers and other special care units

\section{Description:}

This poster will present the approach to institution-wide implementation of advance care planning (ACP) at our tertiary care cancer center.

The Departments of Patient Support and Advocacy and Supportive Care Medicine (SCM) collaborated to lead systematic change in ACP. Support to provide Respecting Choices ${ }^{\circledR}$ training was obtained from executive leadership and an ACP Implementation Task Force was established. To foster understanding and adoption of ACP, an ACP-specific electronic interdisciplinary plan of care was developed and ACP content has been integrated into the annual mandatory education requirements for all Center employees.

The Blood and Marrow Transplant (BMT) Program was an early adopter that restructured its ACP process and increased its advance directive (AD) completion rate from $41 \%$ to $93 \%$. This has contributed to decreased LOS for dying patients, less compassion fatigue among nurses, and fewer ethics consultations.

SCM restructured its processes to prioritize and monitor ACP for all patients. AD status is reviewed for all SCM inpatients and the $\mathrm{AD}$ completion rate has increased from an average of $40 \%$ to $55 \%$.

Overall, a proactive approach was adopted to help patients and families understand, communicate, and document wishes for care consistent with quality palliative care.

52. Preparedness Survey for Joint Commission's Palliative Care Requirement with LVAD Implantation as Destination Therapy

Montefiore Medical Center

Bronx, NY

Team/Contact Information:

Jhosselini A. Cardenas-Mori, MD

Palliative Care Attending

Montefiore Medical Center

$2^{\text {nd }}$ floor

3347 Steuben Avenue

Bronx, NY 10467

jcardena@montefiore.org

Project Team Members:

Elizabeth Chuang, MD

Cecilia Nucci, RN, BSN

Laura Hawks, MD

James Fausto, MD

Topics: Outcomes of interest to payors supporting palliative care service delivery across settings (PCO)

Description:

As of 2012, 101 centers in the United States have been approved to perform LVAD placement as destination therapy. Historically, there has been little regulation over these programs and general practice often may not include the integration of a palliative care team into care provided to LVAD recipients. New regulations set by the Joint Commission require programs desiring Advanced Certification in Disease-Specific Programs to provide an interdisciplinary health care team, including at least one cardiothoracic surgeon, at least one cardiologist (including one heart failure specialist), a registered nurse with experience in $\mathrm{VAD}$, a VAD coordinator (who may be the registered nurse), and a social worker with experience with VAD recipients as well as a palliative care specialist who has expertise working with VAD patients and their families. These new regulations should have taken effect since October of 2014. The new regulations would also mandate detailed discussions about alternative options, risks, and complications of the procedure as well as the provision of extensive supportive services following the LVAD placement. We created a survey with the main objective to evaluate the perception of readiness of palliative care services into the LVAD evaluation process. The survey will be delivery to the palliative teams in the LVAD implantation Joint Commission certified hospitals. This study will be descriptive and our future plan will evaluate learning interventions of the palliative care team in the LVAD evaluation process.

\section{Development of Palliative Care Guidelines in Singapore} National Cancer Centre Singapore

Singapore

Team/Contact Information:

Patricia SH. Neo, MD

Deputy Head, Consultant

National Cancer Centre Singapore

11 Hospital Drive

Singapore, 169610

Neo.S.H@nccs.com.sg

Project Team Members:

Geraldine Kheng Lin John

Angel Onn Kei Lee

Topics: Coalition and Advocacy: Regional and National Palliative Care Initiatives (e.g., statewide coalitions/political advocacy)

Description:

Development of Palliative Care Guidelines in Singapore

PSH Neo, TJ Lo, JKL John, CM Yee, and A Lee.

Background: Despite its renowned health care system, Singapore ranked $18^{\text {th }}$ in the world on the Quality of Death Index. ${ }^{1}$ In response, the Ministry of Health Singapore commissioned a report on the National Strategy for Palliative Care. This report, released in 2011, called for the development of local guidelines in palliative care to reflect core elements of good palliative care for all institutions in Singapore.

Aim: This poster describes the development of the Guidelines for Palliative Care in Singapore.

Method: A literature review was performed by local palliative care experts to develop a draft set of guidelines and corresponding best practice indicators. Subsequently, a panel of health care professionals were consulted on the validity and appropriateness of the draft guidelines through use of a modified RAND Delphi procedure. The guidelines were subsequently subjected to further public and health care stakeholder consultation.

Results: Thirteen guidelines and 51 indicators were identified.

Conclusion: These guidelines have since been endorsed by the Ministry of Health and are currently being implemented across palliative care services in Singapore.

\section{Reference}

1. The quality of death. Ranking end-of-life care across the world. http://graphics.eiu.com/upload/eb/qualityofdeath.pdf (Last accessed August 20, 2015).

\section{The PCIA and Access to Palliative Care in Terminal Patients}

New York Methodist Hospital

Brooklyn, NY

Team/Contact Information:

Kitty E. Victoria, MD

Internal Medicine Resident 
New York Methodist Hospital

492 3rd. Street, \#2A

Brooklyn, NY 11215

theartsinmedicine@gmail.com

Project Team Members:

Sarita Patel, $M D$

Topics: Coalition and Advocacy: Regional and National Palliative Care Initiatives (e.g., statewide coalitions/political advocacy)

\section{Description:}

Background: Studies have shown that over $50 \%$ of end-oflife discussions take place for the first time in the hospital and that terminal patients often have unrealistic views regarding the possible scope of treatment. The Palliative Care information Act (PCIA) was passed in an attempt to address lack of access for terminal patients to palliative care services. A multidatabase systematic review was performed on published studies from 2010 to the present and there were none found measuring the effectiveness of the PCIA.

Objectives: We aimed to study the effect of the PCIA on access to palliative care services.

Methods: We conducted a retrospective chart review of all terminal patients who expired at Kingsbrook Jewish Medical Center from January 2010 to August 2013 in relation to passing of the PCIA.

Results: Pre-law (prior to the law passing) $12.3 \%$ of terminal patients received palliative care consults, $25 \%$ during the transition period (time between passing of law and when it came into effect), and $37.7 \%$ post-law (after coming into effect $),(p<0.001)$.

Conclusions: Legislation has significant effect on terminally ill patient's access to palliative care services and can change the culture of a hospital to be more pro-palliative for the appropriate populations.

55. Education to Increase Palliative Knowledge and Capacity

Niagara Health System

Niagara-on-the-Lake, ON Canada

Team/Contact Information:

Dr. David C. Free

1823 Niagra Stone Road

Niagara-on-the-Lake, ON Canada LOS $1 \mathrm{JO}$

(443) 883-1797

oboedavid@yahoo.com

Topics: Education: Methods of training in primary/generalist palliative care

Description:

Close to 2 million people live in U.S. nursing homes. With an aging population, evidence-based strategies are needed to increase palliative care capacity in long-term care. The focus of this poster is to present the implementation and evaluation of a blended palliative care educational intervention in a 200-bed nursing home in Maryland. An evidence-based practice model and adult learning theory informed project implementation and content delivery. The Palliative Care Quiz for Nursing (PCQN), a validated tool, was used to assess participants' $(n=50)$ baseline knowledge.

During the first of two learning sessions, participants viewed a 30-minute online presentation covering basic palliative care concepts. A week later, a face-to-face review of the concepts was held. Based on androgogy, concepts were made relevant to the unique role of each participant. The result was a 39\% group mean score increase. The participants agreed $(100 \%)$ that the education helped them gain a better understanding of palliative care, would increase referrals, and would improve their ability to provide care. Referral rates and patient outcomes have subsequently improved at the site. These results support the effectiveness of a blended educational approach with evidencebased content informed by adult learning and cognitive theory to increase palliative knowledge and capacity in long-term care.

56. Caring for Vulnerable Geriatric Patients with OASIS North Shore- LIJ Health System

Hofstra North Shore-LIJ School of Medicine

Hyde Park, NY

Team/Contact Information:

Maria T. Carney, MD

North Shore-LIJ Health System

Hofstra North Shore-LIJ School of Medicine

270-05 76th Avenue

Research Building, Second floor

Hyde Park, NY 11040

(718) 470-7270

mcarney@nshs.edu

Project Team Member:

Brian E. Emmert Jr., BS

Topics: Palliative Care Delivery/Operations: Innovative and emerging community-based palliative care models (e.g., home/ office/clinic/long-term care)

Description:

Elder orphans are aged, community-dwelling individuals who are socially and/or physically isolated, without a known family member or designated surrogate. They are at high risk for failure to thrive and loss of independence and safety, which can engender burdens for patients and health systems. Moreover, they are at higher risk for adverse health effects. Using the University of Michigan's Health and Retirement Study, we estimate that the prevalence of this demographic is about $22 \%$ of the over- 65 population, a number expected to increase as the population ages. Thus, provisions must be made for those at risk of becoming elder orphans to appropriately plan for their futures, educate them about community resources, and ensure their safety. We propose the Older Adult Services for Independence and Safety (OASIS) assessment center to care for this and other vulnerable geriatric populations. This multi-disciplinary approach links public-domain community resources with health care delivery. OASIS is dedicated to identifying at-risk older community-dwelling adults, assessing their needs by monitoring health and wellness, and assisting with medical and social care by specializing in a myriad of geriatric assessments. We hope to encourage action to be taken to prepare for the aging population while concurrently raising awareness for elder orphans.

\section{Transforming the Experience of People with Advanced Illness at a Health System}

North Shore-LIJ Health System

Hofstra North Shore-LIJ School of Medicine

New Hyde Park, NY

Team/Contact Information:

Maria T. Carney, MD

Chief, Division of Geriatric and Palliative Medicine

North Shore-LIJ Health System

Hofstra North Shore-LIJ School of Medicine

270-05 76th Avenue

Research Building, Suite B214

New Hyde Park, NY 11040

(718) 470-7270

mcarney@nshs.edu 
Project Team Members:

Tara Liberman, DO

Alexander Rimar, $M D$

Lori Attivissimo, $M D$

M. Isabel Friedman, DNP

Topics: Palliative Care Delivery/Operations: Innovative hospital-based palliative care models

Description:

Using a collaborative structure, 17 teams from inpatient, outpatient, and service line settings developed reliable processes to identify patients with advanced illness, conducted conversations with patients and their families to develop realistic goals of care, ensured services align with patients' needs and preferences, and communicated via documentation through the continuum. the executive directors and medical directors at the involved settings, along with system-level direction, allowed for buy-in and support. A definition of advanced illness was developed and adopted universally throughout the health care system. Using the Model for Improvement as the transformational methodology, identification tools were developed and adopted. Education programs were developed for goals-ofcare conversations by partnering with the Palliative Care Service Line. Once identified, goals of care were documented in the medical record and communication tools were developed to ensure congruence to the next level of care throughout the health care continuum. Evaluation measures being tracked include: the number of patients identified, the number of conversations conducted with documented goals of care, palliative care and hospice referrals, and goals of care hand-offs at the transitions of care. Identification and documentation tools with be presented. Transitional hand-offs to community services will be shared.

58. Advanced Illness Management in the Emergency Department

North Shore University Hospital-North Shore LIJ Health System

Manhasset, NY

Team/Contact Information:

Dr. Tara Liberman

North Shore University Hospital-North Shore LIJ Health System

270-05 76th Avenue

Research Building

3rd floor, Suite. B379

New Hyde, NY 11040

(718) 470-3116

tliberma@nshs.edu

Project Team Members:

Elizabeth Kenjesky, RN

Nancy Kwon, MD

Maureen Hinkelman

Leslie Lindenbaum

Topics: Palliative Care Delivery/Operations: Approaches to palliative care delivery in specific hospital settings (e.g., ICU, ED)

\section{Description:}

Patients with advanced illnesses visit our emergency rooms everyday without ever having had a discussion with their health care providers regarding their goals of care (GOC) and advanced directives (AD). The project goal was to identify patients in the emergency room with a terminal illness, conduct and document a GOC conversation, and, if appropriate, transfer to hospice. An interdisciplinary team (IDT) involving a hospice agency, palliative and emergency medicine was formed to identify advanced illness patients who would benefit from being directly admitted to hospice from the emergency department (ED). From April 2013 to December 2014, 81 patients from the ED were referred directly to hospice. Since $8 / 14$, over $95 \%$ had documented GOC discussion by ED staff. Of these, 35 were directly transferred to an inpatient hospice and 21 to home hospice. Twelve patients refused services. Two went home on home care. Three expired prior to discharge. Eight required admission. All patients admitted had close follow-up by the palliative care team. The majority of these patients died within one week. Patients with advanced illness need good GOC discussions to align with their wishes regarding end-of-life care. The ED can be a place to incorporate discussions and processes.

\section{Risk Factors for Hospital Readmission of Hospice Patients}

North Shore University Hospital

Manhasset, NY

Team/Contact Information:

Diana C. Martins-Welch, MD

Attending Physician

North Shore University Hospital

172 Willis Avenue

Floral Park, NY 11001

dmartins1@nshs.edu

Project Team Members:

A. Wilson

B. Earle

A. Kozikowski

L. Attivissimo

L. Rosen

R. Pekmezaris

Topics: Other

Description:

A retrospective case control study designed to examine potential risk factors for readmission to the hospital within one week of discharge to home hospice. The primary outcome variable was hospital readmission within 7 days. Cases were defined as subjects who were discharged to home hospice and readmitted to the hospital within 7 days. Controls were defined as subjects who were discharged to home hospice and were not readmitted to the hospital within 7 days. Cases were significantly younger than controls (69.5 versus 77.0). Cases were more likely to be Hispanic (15.2 versus 5.1), Asian (15.2 versus 5.1 ), and other (13.0 versus 2.6) when compared with controls. Cases were more likely to speak Spanish (13.3 versus 3.5 ) or other (20.0 versus 5.3) and less likely to speak English (66.7 versus 91.2). Cases were less likely to have Medicare only ( 8.7 versus 82.9) and more likely to have Medicaid (32.6 versus 4.3), private insurance (13.0 versus 10.3), or some other form of insurance. Our data highlight four risk factors, namely age, race, language, and insurance status as factors predicting readmission within 7 days of acute care hospital discharge. Further study of these predictors may identify opportunities for interventions to obviate these readmissions.

60. Systems BRIDGES: Community Outreach for Asian Population

North Shore University Hospital

Manhasset, NY

Team/Contact Information:

Isabella Park

Attending Physician, Palliative Medicine 
North Shore University Hospital

300 Community Drive

Manhasset, NY 11030

ipark1@nshs.edu

Project Team Members:

Maria Carney, $M D$

Bridget Earle, $M D$

Lawrence Lin

Barbara Felker

Mohini Mishra

Miranda Chau

Sung Jae Shin

Topics: Palliative Care Delivery/Operations: Innovative and emerging community-based palliative care models (e.g., home/ office/clinic/long-term care)

Description:

The Asian community is one of the country's fastest growing demographics, and in New York City, Asians are now 12\% of the population. Despite their growing numbers, the Asian community remains vulnerable and underserved, mostly due to language and cultural differences. System BRIDGES was founded by the North Shore LIJ Health System to engage employees who are passionate about building a relationship with this diverse community through shared understanding of spiritual, cultural, and ethical values in the context of health care delivery.

Initially beginning as a hospital-based program to educate health professionals about the Asian community, System BRIDGES moved to community outreach with the help of the Esther Ha Foundation. One area of particular need was in the area of advanc directives, where sessions were conducted for advance care planning.

Additionally, the New York tri-state area has a rapidly increasing Chinese population in the bureau of Queens. Similarly, of the North Shore LIJ Health Plan, Care Connect, $42 \%$ of the Managed Long Term Care (MLTC) patient base is Chinese necessitating the creation of a distinct team of nurses, service coordinators, and sales and outreach staff that speak Mandarin, Cantonese, and Fujianese. As the program expands, new opportunities for outreach become apparent.

61. Analysis of a Palliative Care Unit's Operational Metrics

North Shore University Hospital Palliative Care Unit

Manhasset, NY

Team/Contact Information:

Bridget I. Earle, MD

North Shore University Hospital Palliative Care Unit

15 W 116st, Apt. 4B

New York, NY 10026

(646) 797-9401

BEarle1@NSHS.edu

Topics: Data and Outcomes: Successful use of metrics in community or hospital settings

Description:

North Shore University Hospital has a 10-bed palliative care unit (PCU) with up to six dedicated in-patient hospice beds. We conducted a retrospective review of operational metrics to provide better quality of care. We analyzed discharges from the PCU from February 1, 2014 to January 31, 2015 via the electronic medical record. Data collected included number of patients admitted to the PCU, demographics, disposition, length of stay (LOS) in hospital, LOS in PCU, and days to palliative consultation. There were 705 admissions to the PCU, 487 were palliative and 218 in-patient hospice. Average age for the groups was 75.9 and 74.1 , respectfully. The ratio of male to female was equal and most patients were white. The mortality rate for the palliative was $61.9 \%$ and hospice was $74.9 \%$. The average LOS for the palliative patients was 4.8 days in the PCU and average days to consult was 7.2 days. The patients who were admitted to the PCU under palliative have an increased LOS and days to consult of one week. Although the data continue to be analyzed, our group now has the opportunity to build on the metrics by obtaining palliative consultations earlier in the hospital stay.

\section{Effects of a Self-Care Program in a Palliative Care Setting}

North Shore University Hospital Palliative Care Suite

Manhasset, NY

Team/Contact Information:

Cheryl Gancio, $R N$

North Shore University Hospital Palliative Care Suite

300 Community Drive

Manhasset, NY 11030

BEarle1@NSHS.edu

Project Team Members:

Bridget I. Earle, $M D$

Topics: Palliative Care Teams: How teams work together to maximize team health and avoid burnout

\section{Description:}

North Shore University Hospital's Palliative Care Suite (PCS) specializes in managing and treating symptoms and the emotional needs of patients and families. Approximately $80 \%$ of the admitted patients expire on the unit. This high rate of patient expiration presents a unique challenge for staff. To promote self-care and prevent burnout we implemented a yoga program for the providers in the PCS. The objective of this study was to assess the effects of staff participation in yoga sessions on burnout, compassion fatigue, and self-care. A fellow nurse certified yoga instructor was identified to run each session. Participation waivers were obtained from all staff. The yoga program started in June 2013 once to twice weekly in the PCS. Participants were surveyed to evaluate compassion fatigue, burnout, and perception of organizational involvement using the professional quality of life scale (ProQOL). There were a total of 23 subjects; $13(56.52 \%)$ participated in yoga and $10(43.48 \%)$ did not. Results showed no difference in compassion fatigue. The majority of subjects reported high perception of organizational involvement from their managers after the program was implemented. The program was feasible, of low cost, sustainable, and identified the value of support from management to improve practitioner self-care and burnout.

\section{Hospice Enrollment Changes after Use of AI Tool in} ICUs

North Shore LIJ Medical Group

Manhasset, NY

Team/Contact Information:

Hannah E. Phillips

North Shore LIJ Medical Group

300 Community Drive

Palliative Care Unit

Manhasset, NY 11030

(516) 562-1621

BEarle1@NSHS.edu

Project Team Members:

Bridget I. Earle, MD 
Topics: Palliative Care Delivery/Operations: Approaches to palliative care delivery in specific hospital settings (e.g., ICU, ED)

\section{Description:}

The Advanced Illness: Beds (AIB) Initiative was started to align goals of care in ICUs. Advanced illness (AI) occurs when serious conditions cause health and functioning to decline. AI patients are considered for transfer to the Palliative Care Suite (PCS) after a palliative medicine consultation. ICU patients can be admitted to the PCS as inpatient hospice. This study tracked hospice enrollment in the PCS before and after the AIB Initiative. A retrospective data review was performed on PCS patients discharged from February 1, 2014 to June 30, 2015. Between February 1, 2014 and June 30, 2015, 1,023 patients were admitted to the PCS. Prior to the initiative 705 patients were admitted; 318 after. In the year before, 175 patients were transferred from ICUs, 10 of which were admitted as inpatient hospice. After the initiative, 65 patients were transferred to the PCS from ICUs, 12 of which were inpatient hospice. Prior to the initiative $5.71 \%$ of ICU transfers to the PCS were hospice enrollees. The percentage has since increased to $18.46 \%$. The average number of ICU to hospice patients per month has increased from 0.83 to 2.4 since the initiative, a $190 \%$ increase. Using AI criteria in the ICUs allows for earlier identification, clarification of goals of care, and increased hospice enrollment.

64. Innovation Meets Future Demands: A Novel Approach to Fellowship Training in Hospice and Palliative Medicine North Shore LIJ Medical Group

Manhasset, NY

Team/Contact Information:

Sindee Weiss-Domis, MD

Program Director, Palliative Medicine Fellowship

North Shore LIJ Medical Group

300 Community Drive

Manhasset, NY 11030

(516) 562-8825

Sweiss3@nshs.edu

Project Team Members:

Bridget Earle, $M D$

Topics: Other

Description:

North Shore University Hospital has a growing Hospice and Palliative Medicine (HPM) Fellowship training program, established in 2007 and accredited in 2009. As the number of people with serious medical illness increases, the need for hospice and palliative medicine trained physicians has increased. Additionally, providers can no longer sit for the certification exam using verification of clinical experience. Review of the Accreditation Council for Graduate Medical Education's frequently asked questions notes that HPM fellowship programs have the flexibility to accept fellows with the expectation of completing the program on a part-time basis over the course of 24 to 36 months. ${ }^{1}$ As a result; we are piloting a part-time fellowship in Hospice and Palliative Medicine that commenced July 2015. The primary boards were contacted and they agreed in writing to allow entrance to the certifying exam upon successful completion of all program requirements over a 24month period. We anticipate this will be a viable alternative to full-time training, allowing more providers the acquisition of subspecialty competencies and board certification in hospice and palliative medicine.

\section{Reference}

1. https://www.acgme.org/acgmeweb/Portals/0/PDFs/FAQ/540_ hospice_and_palliative_medicine_FAQs_07012015.pdf (Last accessed March 1 to May 4, 2015.)

\section{Palliative Care for Inpatient COPD Patients}

Olathe Medical Center

Olathe, KS

Team/Contact Information:

Jana L. Braklow, NP

Olathe Medical Center

2341 W. Fredrickson Drive

Olathe, KS 66061

(913) 791-3520

jana.braklow@olathehealth.org

Project Team Members:

Lufei Young PhD, RN, APRN-NP

Topics: Palliative Care Delivery/Operations: Approaches to palliative care delivery for specific population types (e.g., heart failure/cancer/dementia/frailty)

\section{Description:}

The current practice problems existing in acute care settings regarding the care of COPD patients include: 1) inadequate symptom management; 2) incongruent care between inpatient and outpatient care settings; 3) lack of knowledge, skills, and experience in providing palliative care in general practitioners; and 4) unmet needs and suboptimal quality of palliative care in COPD patients.

To address these issues, we have developed a clinical framework in which the COPD palliative care services are integrated into the standard of care in the acute care setting. The disease management core addresses the current standard of care in managing COPD patients during acute exacerbation or maintenance phases. Crisis management and disease modification protocols are utilized in respective phases. The palliative care core is primarily focused on symptom management, including the assessment and management of four intertwined symptoms (i.e., dyspnea, impaired functioning, depression, and anxiety). The symptom management is guided and supported by patient-centered goals of care and end-of-life planning. The education core includes patient, caregiver, and provider education programming. The center of the framework is the transitional care core that addresses the key elements linking efficient and effective communication strategies, advance care planning, and decision aids.

\section{Building the Business Case for Ambulatory PC Clinic} Oregon Health \& Science University

Portland, OR

Team/Contact Information:

Linda L. Blakely, MS

Research Assistant

Oregon Health \& Science University

3181 SW Sam Jackson Road

Portland, OR 97239

(503) 805-7817

frommee@ohsu.edu

Project Team Members:

Brie N. Noble, BS

Brad Cota, MBA

Melissa Alvarado, BS, CTR

Erin Watson, BS, RHIA, CTR

Erik Fromme, MD, MCR, FAAHPM 
Topics: Palliative Care Teams: Demonstrating clinical and financial outcomes for team roles

\section{Description:}

This poster summarizes our efforts to devise a sustainable business plan for an outpatient palliative care clinic housed in Oregon Health \& Science University's Knight Cancer Institute. We will share what we've learned in three key areas: 1) structuring the clinic, 2) choosing metrics, and 3) working with our organization and with payors. Preliminary analysis of metastatic cancer patients shows lower per-patient hospital costs in the last 6 months of life for outpatient palliative care clinic patients than for a control group.

\section{Effectiveness of a Mobile Mentoring PC Team}

Palliative Care Development Associates

Evergreen, $\mathrm{CO}$

Team/Contact Information:

Nancy A. Robertson, NP

PCDA NP Mentor

Palliative Care Development Associates

5981 Bluebell Ln.

Evergreen, CO 80439

(720) 312-1256

nancy.robertson@pallicareda.net

Project Team Members:

Bob Davidson, LCSW

Topics: Other

Description:

Significant hurdles face the development and sustainability of community-based palliative care programs. Workforce shortages of qualified clinicians with expertise in palliative care head the list of major obstacles. In an effort to meet this clinician deficit, a novel pilot program was developed. A mobile mentoring team comprised of a palliative care certified NP and an experienced palliative care LCSW/Chaplain was created. This team joined forces with two community hospice programs with the goal of addressing this challenge.

Adhering to national standards and guidelines of care, the NP mentor paired with the inexperienced NPs. Using standardized curriculum, monthly mentoring meetings and full phone access, the NP mentor championed the mission to increase the new NPs' PC knowledge from the beginning. The LCSW/Chaplain mentor worked with inexperienced social workers and chaplains, providing numerous educational meetings, coaching, and support. In addition, joint sessions were conducted by both mentors with the palliative care team members to address role differentiation, foster true IDT relationships, and to review case studies.

The results indicate that the combined efforts of a mobile mentoring team with community organizations is a successful model to meeting the overwhelming demand for qualified palliative care clinicians in the community.

\author{
68. Initial Impact of Rural Community Palliative Care \\ Palliative Care of St. Lawrence Valley \\ Potsdam, NY \\ Team/Contact Information: \\ Marie-Paule (Missy) Heylen, NP,ACHPN, ANP-c \\ Palliative Care of St. Lawrence Valley \\ 6805 UD Hwy. 11 \\ Potsdam, NY 13676 \\ (315) 265-3105 \\ mheylen@hospiceslv.org \\ Project Team Members: \\ Diane Pickering, $R N$
}

Topics: Data and Outcomes: Successful use of metrics in community or hospital settings

\section{Description:}

A community palliative care program was initiated by a hospice in January 2015 in Rural St. Lawrence County. The palliative care team, consisting of a physician, nurse practitioner, social worker, and RN, served a total of 139 patients in 18 months. The project obtained privileges for the nurse practitioner at four rural hospitals. The hospitals all agreed to subsidize part of the cost. Nurse practitioner, physician, and LCSW visits were billed to Medicare and private insurance. Referrals came primarily from hospitals and physician practices, with referrals also being made by families, home health agencies, and the patients themselves. Most patients were very ill and were seen at home. Patients were followed up through hospital medical record systems. The results demonstrate a positive impact of the program in terms of (1) reduced 30-day hospital readmissions, (2) reduced emergency department visits, (3) reduced hospital admissions, and (4) appropriate and timely referral to hospice services. Results were tracked by hospitals and by major diagnostic groups, showing variation in the results. Outcomes indicate a clear impact on reducing hospitalizations for COPD and cancer patients, but no impact on $\mathrm{CHF}$ emergency department use.

69. Charting the Sacred: Palliative Care Chaplaincy

Pomona Valley Hospital Medical Center

Pomona, CA

Team/Contact Information:

Francisco V. Munoz, MA

Pomona Valley Hospital Medical Center

1282 Hillsdale Drive

Claremont, CA 91711

(909) 630-7697

francisco.munoz@pvhmc.org

Topics: Data and Outcomes: Using technology to improve efficiency and outcomes

Description:

This poster will present the three-stage development of an electronic charting system designed to capture the data from "sacred" encounters between palliative care patients and palliative care chaplains in a manner that provides clinically relevant information for the palliative care physician, nurse, and social worker.

\section{Use of Volunteers in Hospital-Based Palliative Care}

Presence St. Mary's Hospital

Kankakee, IL

Team/Contact Information:

Peter D. LaMotte, LCSW, ACM, ACHP-SW

Regional Director Palliative Care

Presence St. Mary's Hospital

$500 \mathrm{~W}$ Court Street

Kankakee, IL 60901

(815) 937-2125

peter.lamotte@presencehealth.org

Project Team Members:

Ronda Bond

Sue Giacchino, DNP, APN

Lynn McDonald, MD, FACP

Topics: Palliative Care Delivery/Operations: Innovative hospital-based palliative care models

Description:

The Presence St. Mary's Hospital palliative care practitioners were encountering a number of patients in the hospital with no 
visitors, little to no social interaction, and, at times, going through the dying process alone. The hospital's Volunteer Services Department was approached for interested members who might be willing to sit with patients. Discussions ensued and a vision was created to develop a program that now has evolved into an abbreviated ELNEC training for volunteers interested in providing services to our hospital-based palliative care patients. Carts were developed that have numerous items including oils for massage, music, kits for making hand prints, snacks, and bereavement reading materials. The volunteer program has even expanded to assisting with follow-up phone calls and monitoring patient/family satisfaction. Although initially developed for patients that were socially isolated, the program has become extremely popular among the volunteers serving all palliative care in-patients providing services Monday through Friday and on-call. Poster would explain time line of program, program implementation, and how patients are identified for volunteer interaction.

\section{Community Engagement Efforts in Advance Care} Planning

ProHealth Care

Waukesha, WI

Team/Contact Information:

Melissa G. Hale, MSW

Advance Care Planning Coordinator

ProHealth Care

721 American Avenue, Suite 508

Waukesha, WI 53188

Melissa.Hale@phci.org

Topics: Palliative Care Delivery/Operations: Innovative hospital-based palliative care models

\section{Description:}

Advance care planning is a process of understanding, reflecting on, and discussing future medical decisions, including end-of-life wishes. With advancements in nearly every field of medicine in the past century patients are living longer; Yet patients and medical providers alike are often caught unprepared for their end of life or emergent medical situations. ProHeatlh Care has identified advance care planning as a strategic initiative and is working toward providing comprehensive advance care planning education across the continuum of care; from hospital to primary care to community setting.

ProHealth Care, serving patients of all ages in Waukesha County, has set a strategic initiative to educate the Waukesha community on the importance of advance care planning. With partnership from Honoring Choices Wisconsin, PHC has delved into the community offering several programs and services. Such programs include partnerships with local assisted living facilities, the Kwanis Club, the Hispanic Resource Center, and local faithbased organizations as well as community education classes.

\section{Goal Setting in Primary Care (SAGE Program)}

ProHealth Physicians Inc.

Farmington, CT

Team/Contact Information:

David Russell, MD

ProHealth Physicians Inc.

950 Yale Avenue

Wallingford, CT 09492

(203) 265-6900

drossrussell@prohealthmd.com

Project Team Members:

Patty Harris APRN
Topics: Palliative Care Delivery/Operations: Innovative and emerging community-based palliative care models (e.g., home/ office/clinic/long-term care)

\section{Description:}

SAGE (Sensible Assistance with Goals Evaluation) is a palliative program of ProHealth Physicians, a large (300+ provider) Primary Care group and ACO in Connecticut. Patients with lifelimiting illness or advanced frailty are identified and referred by their PCP or care manager. We contact the patient and family to arrange a home visit with the patient and key family support with our trained RN/APRN. The objective is to review current status and establish and communicate goals of care consistent with the patient's condition and wishes. When appropriate, referral to hospice can be initiated, advance directives completed, and DNR arrangements made with the PCP's support. When necessary, follow-up visits or phone contact is maintained until the goals of care are established and are being implemented.

Outcomes under evaluation are rates of hospice utilization, median hospice length of stay, cost of care in the period prior to death, and patient and family satisfaction.

Target penetration is to reach at least $1 \%$ of our Medicare population (c 300 patients) each year. This is intended as the first stage of a more comprehensive program of home support for the patients with palliative needs prior to hospice eligibility.

73. Intensive Care Unit/Palliative Care Educational Videos Providence St. Peter Hospital

Olympia, WA

Team/Contact Information:

Sarah Merrifield, MD

Providence St. Peter Hospital

413 Lilly Road NE

Olympia, WA 98506

(360) 486-6402

sarah.merrifield@providence.org

Topics: Education: Examples of primary palliative care education approaches to address the workforce shortage

Description:

We created five online videos for intensive care unit (ICU) staff at our hospital. The videos address palliative care related topics that were identified by an initial needs assessment survey of ICU staff and through ongoing close collaboration between our ICU and palliative care teams. The video format was selected to overcome barriers specific to educating ICU staff and to optimize participation in primary palliative care education. Funding was through a grant from our hospital foundation, which allowed us to hire a professional videographer. Video topics include: "Palliative Care, Hospice, and the CCU," "Compassion Fatigue in the Critical Care Unit," "Conflict and Moral Distress in the CCU: Communication Strategies that Work," "Death and Dying in the CCU: Opportunities for Healing When There Is No Cure," and "Tracheostomies, Feeding Tubes, and Code Status: Discussing Treatment Options in the Context of Family/Patient Priorities." We wrote quiz questions for each video, and staff can get education credits for watching the videos. Initial informal feedback has been very positive. We plan to measure viewership data of the videos and assess their impact on our ICU culture by using before and after surveys.

74. Using Accessibility of Email to Address Grief and Loss Rady Children's Hospital San Diego

San Diero, CA

Team/Contact Information:

JoAnne Auger, RN

Rady Children's Hospital San Diego 
13127 Via Del Toro

Poway, CA 92064

(858) 966-1700, x6189

jauger@rchsd.org

Project Team Members:

Kimberly Bower, MD

Deborah Schiff, MD

Topics: Palliative Care Teams: How teams work together to maximize team health and avoid burnout

Description:

It is difficult to provide a timely debrief for clinicians following a patient death in the acute care setting, due to conflicting schedules, staffing demands, and the logistics of patient care. Using the concepts behind the narrative medicine movement, that writing can help clinicians as they process of feelings of loss, the team email debrief offers a moment to pause and remember before moving on to tend to another patient's needs. After the loss of a pediatric oncology patient, an email detailing the patient's end-of-life course is written by the Supportive Care RN Coordinator and shared with the entire interdisciplinary care team. The goal of the team email debrief is to work toward closure and peace, both by summarizing the patient and family's final journey, and acknowledging the hard work of the team and the burden of the loss.

75. Getting to Satisfaction: Collecting and Interpreting Referrer Satisfaction Data

Rainbow Kids Palliative Care, Primary Children's Hospital

Salt Lake City, UT

Team/Contact Information:

Dominic Alexander Moore, MD

Rainbow Kids Palliative Care, Primary Children's Hospital

2382 E Beacon Drive

Salt Lake City, UT 84108

(801) 662-3770

dominic.moore@hsc.utah.edu

Project Team Members:

Mary-Ann Bowman, LCSW PhD

Toni Sherwood, NP

Joan Sheetz, MD

Topics: Data and Outcomes: Successful use of metrics in community or hospital settings

Description:

Rainbow Kids Palliative Care Team covers all inpatient units 5 days a week and maintains phone contact with patients after discharge. Referrals are generated by the primary medical team caring for the patient with no current trigger tool or checklist. In designing our survey we found little published regarding referring clinician perception of palliative care services. We sought to design a useful tool, collect actionable data, analyze results, and reflect on how this process might be improved. One hundred twenty-eight responses were received; $85 \%$ had referred to PCC in the past; $93.3 \%$ agreed/strongly agreed that PCC benefits patients; $71.1 \%$ agreed/strongly agreed that PCC makes useful recommendations for care after discharge; $63.5 \%$ felt PCC made useful recommendations for symptom management; $61 \%$ indicated that PCC involvement improved provider understanding of goals of care; $52 \%$ of those responding had been supported emotionally by the PCC team; $39.8 \%$ felt that PCC involvement had caused conflict among patients' care teams. Based on the data obtained the team was able to identify high-priority issues and undertook PDSA cycles to address concerns with documentation and interaction with referrers. As with any measure of satisfaction, we found that some opinions of best practice among referrers were different and at times conflicting.

76. Pharmacist Involvement in the Palliative Care Clinic

San Antonio Military Medical Center

Fort Sam Houston, TX

Team/Contact Information:

Emily Davies, PharmD

San Antonio Military Medical Center

7601 Gateway Blvd., Apt. 1836

Live Oak, TX 78233

(210) 539-8608

Emily.e.davies3.civ@mail.mil

Project Team Members:

Pamela Moore PharmD, BCPS, CPE

Teresa Albanese, PhD

Steven Radwany, MD

Topics: Palliative Care Teams: Staffing the interdisciplinary team including staffing benchmarks and maximizing the roles of pharmacists/social workers/chaplains/nursing and medicine.

\section{Description:}

The objective of this study was to describe the role of the clinical pharmacist in an interdisciplinary outpatient palliative care clinic (OPPCC) serving both cancer and non-cancer patients at Summa Health System Akron City Hospital (ACH), and to document and analyze the clinical pharmacist's functions and interventions for all patients reviewed by the pharmacist over a 10 -month period. A quality improvement project reviewed and granted exemption by ACH IRB. Data collected involved patient demographics, pharmacist recommendations and interventions. Data were analyzed using descriptive statistics. Over the 10 months, the pharmacist was involved with 180 visits, affecting 80 different patients. Medication-related interventions by the pharmacist totaled 456 , with $44 \%$ of these interventions directly involving medication regimen changes and reconciliations. Nineteen percent of the interventions included both patient and provider education. Overall, the pharmacist played a significant role in the OPPCC. Pharmacist recommendations to providers involving medication changes were accepted in the majority of patient visits (456 interventions during 180 visits). There were discrepancies found and corrected in $82 \%$ (53/65 medication reconciliations) of pharmacistconducted medication reconciliations.

\section{No Time to Spare: Early Palliative Care in the Safety} Net

San Francisco General Hospital

San Francisco, CA

Team/Contact Information:

Anne Kinderman, MD

Director, Supportive \& Palliative Care Service

San Francisco General Hospital

1001 Potrero Avenue

Box 0862

San Francisco, CA 94110

(415) 206-3303

anne.kinderman@ucsf.edu

Project Team Members:

Heather A. Harris, MD

Leslie Safier, $M P H$

Topics: Palliative Care Delivery/Operations: Approaches to palliative care delivery for specific population types (e.g., heart failure/cancer/dementia/frailty) 


\section{Description:}

Studies suggest that under-insured patients are more likely to present with later-stage cancers than Medicare-insured patients, and they may be more likely to be hospitalized urgently for cancer care. However, studies of palliative care (PC) interventions for cancer patients suggest that the greatest impact can be achieved if these services begin "early" ( $\geq 90$ days prior to death). To determine the appropriate model for providing early palliative care for cancer patients in our system, we examined where patients receive their initial cancer diagnosis, and at what stage they are diagnosed. We used data from the hospital's tumor registry to identify cancer stage and location of diagnosis, over a 5-year period. Our results showed that of the 2928 patients diagnosed with cancer, 26\% $(n=759)$ were diagnosed while hospitalized. Patients who were diagnosed while hospitalized were more likely to be diagnosed with stage 3-4 disease (49\%, $n=372)$, versus stage $0-1(21 \%, n=163)$. Patients who received their diagnosis in the outpatient setting were more likely to be diagnosed with stage $0-1(40 \%, n=865)$, versus stage 3-4 $(32 \%, n=688)$. These results suggest that "early" palliative care for cancer patients in our hospital must often involve initial consultation while patients are hospitalized, with prompt outpatient follow-up.

78. The Forgotten Organ Donor: A Call for Greater Palliative Care Involvement

San Francisco General Hospital \& Trauma Center

San Francisco, CA

Team/Contact Information:

Heather A. Harris, MD

San Francisco General Hospital \& Trauma Center

Box 8062

San Francisco, CA 94110

(415) 206-3786

Heather.Harris@ucsf.edu

Project Team Members:

Nikole Neidlinger, $M D$

Topics: Palliative Care Delivery/Operations: Approaches to palliative care delivery for specific population types (e.g., heart failure/cancer/dementia/frailty)

Description:

Organ donation has evolved over the past 40 years as optimal therapy for patients with end organ disease. Recent efforts have increased available organs, particularly through the expansion of donation after cardiac death (DCD), yet great need remains with an estimated 18 patients dying per day while awaiting an organ. Just as in other instances of end-oflife care, palliative care programs have expertise that may enhance the care of donors, surrogates/family, and health care providers, but palliative care is not yet routinely integrated. It may be argued that expertise in communication around end-oflife decision making and complex medical concepts such as brain death or donation after death has been declared by cardiopulmonary criteria, expert symptom management for actively dying patients, and a holistic, interdisciplinary focus on family support and bereavement would particularly benefit these end-of-life situations.

This poster will address:

1. criteria for becoming a deceased organ donor and review clinical tools used for predicting possible DCD candidates

2. review processes of organ donation for deceased donors
3. best practices in interfacing with organ procurement agencies and conversations with surrogates about organ donation

4. review the literature base demonstrating significant palliative care needs for surrogates in these situations, and

5. how some palliative care programs have integrated themselves into standard care

79. Creating an Acuity and Productivity Measurement Tool for an Inpatient Palliative Care Service

Santa Monica UCLA Medical Center

Santa Monica, CA

Team/Contact Information:

Jeannette Meyer, RN, MSN, CCRN, CCNS, PCCN, ACHPN

Santa Monica UCLA Medical Center

19350 Sherman Way

Unit 105

Reseda, CA 91335

(424) 259-8161

jmeyer@mednet.ucla.edu

Topics: Palliative Care Delivery/Operations: Innovative hospital-based palliative care models

Description:

The Palliative Care CNS and Physician Faculty identified the need to create a tool to measure the acuity of palliative patients at an academic medical center. This tool assesses factors such as common patient diagnoses and requirements, average time of visits, and whether daily visits were required. This tool has also demonstrated value in measuring the productivity of palliative team members.

80. Staffing for a Small, Rural Palliative Care Program Schneck Medical Center

Seymour, IN

Team/Contact Information:

Sally B. Acton, BSN, RN, OCN, MSM

Director, Cancer and Palliative Care Services

Schneck Medical Center

9482 W Co. Road $200 \mathrm{~N}$

Norman, IN 74264

(812) 522-0474

sacton@schneckmed.org

Project Team Members:

Donna Butler, MSN, ANP-BC, OCN, ACHPN, FAAPM

Dana Prieto, BSN, $R N$

David Hartung, DO

Leighana Crenshaw, MSW, LSW

Anita Collins, MSN, FNP-BC,

Rev. Stephen Barrett

Colette Mills, BSN, RN, CHPN

Aaron Banister, PhD

Topics: Other

Description:

Schneck Medical Center integrated the objective "to recognize and grow nurse practitioners (NP) to further assist in the development of service lines and quality of care issues" into its Strategic Plan. An action plan was implemented in 2013 with a 2-year time line. The plan included the approval of a workforce classification policy entitled Critical Need Student Position. NPs were the first to be designated as a Critical Need Student Position. SMC partnered with the IUPUI School of Nursing to 
develop a Cohort Program. Ten clinical nurses were accepted into the program. Full tuition was paid and scholarships given to offset books and fees. Each NP student was paid as a full-time employee, required to work a maximum of six shifts a quarter, and fulfill a 4-year commitment post-graduation. The outcome would see the number of org-wide NPs increase by $100 \%$. The Palliative Care Program at SMC began as an outpatient clinic in the Cancer Center with one NP. Since moving into the inpatient realm, the department needed another NP to fulfill the needs of the COPD and CHF population. Palliative Care was able to obtain a full time NP from the Cohort Program, beginning October 1

\section{Transforming the Transdisciplinary Model}

Seattle Children's Hospital

Seattle, WA

Team/Contact Information:

Cheryl Arnett, MHA

Seattle Children's Hospital

4800 Sand Point Way NE

MB.5.605

Seattle, WA 98105

(206) 987-4719

cheryl.arnett@seattlechildrens.org

Topics: Palliative Care Delivery/Operations: Innovative pediatric palliative care programs including combined adult/pediatric models

\section{Description:}

Objectives: Identify how to expand the scope of service and decrease compassion fatigue.

Background: Compassion fatigue can lead to unexpected sick days and turnover. The risk of compassion fatigue for the Transdisciplinary Model needs to be considered when evaluating the effectiveness of this model.

Case Description: The Transdisciplinary Model of care started in 2001 in the Pediatric Palliative Care Consultation Service at Seattle Children's where one clinician was assigned to a patient, regardless of their clinical background. In 2014, compassion fatigue was at an all-time high, and leadership was compelled to change the model or risk losing members of the team to burnout. The Hybrid Transdisciplinary Model of care was implemented where the burden of a case was shared between a non-physician clinician and an Attending. Six months after changing the model of care, staff vacations were at the lowest point in 2 years.

Conclusion: The Hybrid Transdisciplinary Model reduces compassion fatigue as evidenced by a significant decrease in vacation usage. Future research is needed to determine if the Hybrid Transdisciplinary Model of care is sustainable and should be idealized while we are in a climate of strategizing how to function with limited resources.

82. Outcomes Data for a Healthcare System: Helping Make the Case for Palliative Care

Sentara Healthcare

Virginia Beach, VA

Team/Contact Information:

Parag Bharadwaj, MD

Sentara Healthcare

4049 Church Point Road

Virginia Beach, VA 23455

(757) 277-6050

paragbharadwaj@hotmail.com
Project Team Members:

Karen M. Helfen

Leo J. DeLeon

Douglas M. Thompson

Jennifer R. Ward

John Patterson

Sriram Yennu

Joe Kim

Kathie S.Zimbro

J. Brian Cassel

Aaron D. Bleznak

Topics: Palliative Care Delivery/Operations: Innovative hospital-based palliative care models

Description:

The process and methodology of delivering outcomes data at a 12-hospital healthcare system will be presented. Data from 7 hospitals on the same EMR will be shared. Common pitfalls, overcoming them, and optimizing for accuracy will be discussed. Methods to optimize database for both internal and external reporting (CAPC Registry) will be highlighted. Strengths and weaknesses will be discussed.

Five studies were done. Their results are summarized:

1. One-year outcomes data of a newly established program at one of the community-based hospitals. Patients were severity adjusted.

o If patients were seen by palliative care within the first 48 hours $(n=353)$ versus later, the difference in length of stay per patient was 5.08 days. This led to a difference of $40 \%$ of the cost per case per day.

o The mortality of patients seen within the first 48 hours versus later in the hospitalization was 1.01 versus 1.1 , respectively.

o The 30-, 60-, and 90-day readmissions dropped significantly after a palliative care consult by $61.5 \%, 47 \%$, and $42.1 \%$, respectively.

o An increase in hospice referrals was noted.

2. A 7-hospital modified matched pair study was performed for patients with sepsis across the system (annualized from a 6-month dataset).

o There was an average difference of 1.67 days in length of stay of patients seen by palliative care within the first 48 hours of admission versus those not seen by palliative care. As an aggregate, palliative care patients in the ICU with sepsis who were seen within the first 48 hours had a shorter length of stay of 1.12 days.

3. System financial impact: Patient/family-directed care plan change methodology (annualized from a 2-month dataset). Whenever a change was made to a more conservative treatment plan, the palliative care clinician, in real time, used a tab on the palliative care navigator developed in the EMR. On the back end, the system picked out the difference in the cost of care pre- and post-change in treatment plan.

o Patient/family directed care plan change methodology to capture the drop in cost of treatments for the hospitals showed an overall cost avoidance that was 1.5 times the total cost of the programs to the system. Each program more than recovered its costs through cost avoidance using this methodology.

4. A 4-month ICU pilot with a full-time physician in one of the hospitals. 
o The same patient/family-directed care plan change methodology in the ICU yielded an annualized cost avoidance of more than $\$ 600,000$ (annualized form 2 moth dataset) and 315 (annualized from a 4month dataset) saved ICU days.

o Other outcomes were measured-increase in referrals, nature of interventions, staff satisfaction, and referrals to hospice.

5. System-wide referrals to hospice (3-month dataset).

o On average, $15 \%(11.5 \%-32.3 \%)$ of all palliative care patients discharged from the hospitals were discharged with hospice care. Of all patients discharged with hospice care, $69.3 \%$ were referred by palliative care.

A recent trend in health care is for multiple hospitals to join to form a system. This calls for the case for palliative care to be made at both the hospital and system level. This comes with its own advantages and challenges.

Through this presentation, we would like to highlight, for our colleagues, the journey of gathering and processing data to show the advantages of palliative care by using outcomes data. This was done in 7 hospitals in which palliative care was established and developed differently. By developing a strategy involving various entities within and outside the system, the best case was developed to validate the current programs and initiate conversations at the system level to invest in its growth. The process and methodologies will be helpful to single-site programs and system-based programs in the country. We hope that the data in this presentation will help support programs that do not have the tools and resources to analyze outcomes data and provide the right tools to the ones that have them.

83. Integrating Palliative Care Medicine into Skilled Nursing and Long-Term Care

Sentara Medical Group Palliative Care Medicine

Norfolk, VA

Team/Contact Information:

Carol D. Miller, NP

Sentara Medical Group Palliative Care Medicine

826 Jerryville Street

Chesapeake, VA 23322

cdmille3@sentara.com

Project Team Members:

Denise Miller, MSN, FNP

Donna Baldassare, DO

Mary Trosien, MSN, FNP

Susan Reutzel, MSN, FNP

Francina Singer, LCSW

Parag Bharadwaj, MD

Topics: Palliative Care Delivery/Operations: Integrating palliative care services (e.g., across a health-system/patient-centered medical home/assisted living/nursing homes)

Description:

Aim: to determine the impact of providing continuity for palliative care medicine (PCM) from the inpatient setting to skilled nursing (SNF) and long-term care facilities (LTC).

Intervention: A full-time PCM nurse practitioner (NP) was hired to change the static care model of discharge without continuity to a dynamic state. This allowed for the PCM NP to unfreeze the current system and promote continuity between the inpatient setting and SNF and LTC. Spragen's software was used to capture data based on the CAPC rounding tool.

Results: A favorable trend on the impact of daily ESAS screening was observed. The avoidance of nine 30-day hospital readmissions, 13 admissions, 19 hospice referrals, and 15 deaths followed by PCM resulted in decreased mortality within the hospital over a span of 5 months. Based on the impact of this initiative, the health care system is investing in replicating this model in other hospitals.

Conclusion: Results suggest that PCM has had a positive impact on providing continuity and symptom management, resulting in a decrease in unnecessary hospital transfers, decrease in hospital mortality, and improved patient outcomes. Further studies are needed to validate findings.

\section{Is Compassionate Pain Management Measurable?}

Shawnee Mission Health

Shawnee Mission, KS

Team/Contact Information:

Cindy Kuklenski, RN

Pain Management/Palliative Care Services

Shawnee Mission Health

$9100 \mathrm{~W} 74$ th Street

Shawnee Mission, KS 66204

(913) 676-2224

cynthia.kuklenski@shawneemission.org

Topics: Education: Targeting Education: Pain management and communication skills

\section{Description:}

In a 500-bed community hospital, a team of nurse educators and the pain management resource nurse developed a competency surrounding RN pain management communication skills. Because pain is a unique symptom that is often associated with fear, anxiety, suffering, and emotions, we wanted to ensure that nurses were delivering pain management care with an emphasis on compassion.

In a simulation lab, RNs were required to demonstrate a comprehensive, compassionate pain assessment, intervene with the appropriate care, then communicate with the (simulated) patient in a manner that relayed empathy, concern, and compassion.

One of our greatest challenges was in designing a method to "measure" RN behavior. Compassion is difficult to measure; it is often regarded as intangible or indefinable. The team created a tool outlining specific criteria that nurses were required to complete during their simulation; numeric values were placed on each criteria (behavior), then the sum total was translated into a pass/fail grade. Other materials included several preworkshop materials to review, a quiz, and a post-workshop evaluation tool. There were $410 \mathrm{RNs}$ who completed the competency.

Outcome data were based on pre- and post-HCAHPS patient satisfaction scores. This poster will present the tools utilized and the outcomes data.

\section{The Family Meeting: A "Procedure" for Medicine Residents}

St. Joseph's Hospital and Medical Center

Phoenix, AZ

Team/Contact Information:

Jeanette Boohene, MD, FACP

Medical Director Palliative Care

St. Joseph's Hospital and Medical Center

12201 N 68th Place

Scottsdale, AZ 85254

jboohene@hotmail.com

Project Team Members:

Jaya M. Raj MD, FACP 
Topics: Communication: Communication best practices (e.g., teaching goals of care conversations/advance care planning/ family meetings)

Description:

To improve palliative care (PC) in our ICUs, our health care system has implemented a version of the IPAL-ICU, Care and Communication Bundle, which requires an interdisciplinary family meeting by day 5 of a patient being in the ICU. This was the ideal opportunity to teach our medicine residents how to effectively facilitate family meetings.

In collaboration with the medicine residency program director, we implemented a curriculum, including a communication lecture with role-playing, "a cheat sheet" highlighting the key points in facilitating a meeting, a requirement for residents to facilitate up to two family meetings during their ICU rotation, under direct observation of a supervising physician, or a provider from the PC team and $\log$ this like all other procedures. An evaluation form rating the resident's performance in specific communication skills is completed by the observer, with feedback to the resident from all members of the interdisciplinary team (IDT) present for the meeting. The resident also completes a self-assessment.

The residents report increased level of comfort facilitating family meetings, appreciation of the value of early family meetings for patients in the ICU, the importance of avoiding medical jargon, and the value of having members of the IDT involved in family meetings.

\section{Primary Palliative Care in Action-Meet Our "Champions" \\ St. Joseph's Hospital and Medical Center \\ Phoenix, AZ \\ Team/Contact Information: \\ Jeanette Boohene, MD, FACP \\ Medical Director Palliative Care \\ St. Joseph's Hospital and Medical Center \\ $12201 \mathrm{~N}$ 68th Place \\ Scottsdale, AZ 85254 \\ jboohene@hotmail.com \\ Project Team members: \\ Diane King RN, MBA/HCM, CNRN, CCRN, ATCN}

Topics: Education: Examples of primary palliative care education approaches to address the workforce shortage

Description:

Our 570-bed hospital, with 100 ICU beds, has a palliative care (PC) team of four providers, seeing consults throughout the hospital. With $52 \%$ of consults in the ICU, it is obvious that our patients palliative care needs cannot be met solely by this team.

The need to educate our bedside nurses in delivering primary palliative care and recognizing when there is a need for a formal palliative care consult has been championed by ICU nursing leadership.

A system-wide initiative using triggers for PC consults was the platform for involving bedside nurses in this endeavor. A PC committee, led by "nursing champions" from each ICU, with input from other IDT members and the PC medical director, is leading the way.

Nursing leadership has been supportive of each ICU sending one "champion" for ELNEC training, to facilitate education on their unit. Each quarter, a topic is prepared by one of the "champions" and displayed on the PC board in each ICU. Bedside nurses are encouraged to read the board and sign off once completed. The next phase is to obtain feedback from our nurses about the value of this education and to extend this to the oncology and medical/surgical units.
87. Association of Cancer-Related Fatigue with Other Symptoms and Quality of Life

Tata Memorial Centre

Mumbai, India

Team/Contact Information:

Arunangshu Ghoshal, MD

Tata Memorial Centre

Parel

Dr. E Borges Road

Mumbai, India

arun.bata@yahoo.com

Project Team Members:

A. Damani

M.A. Muckaden

Topics: Other

Description:

Fatigue with other symptoms affects quality of life (QOL) in advanced cancer patients. This study looks into the factors associated with fatigue, its impact on QOL at baseline, and predictors of improvement of same at first follow-up visit. A prospective, observational study was conducted from January to June 2014. Registered adult advanced cancer patients with the PC clinic meeting the inclusion criteria were assessed after giving informed consent for symptom burden (ESAS) and QOL (EORTC-QOL PAL15) along with demographic details. They were given standard treatment for those symptoms. They met the PC team or were telephonically contacted for the same domains after an interval of 15 to 30 days. Descriptive statistics, comparison of baseline and follow-up data, correlation and multiple linear regressions between fatigue and symptoms at baseline, and logistic regression model to determine factors associated with improvement in fatigue were performed. A total of 402 subjects were assessed at baseline and follow-up (median age, 52 years; $51.6 \%$ male). Significant change in fatigue score was observed at follow-up. Hemoglobin, albumin levels, type of cancer, sites of metastasis, ECOG score, body weight, ESAS items except drowsiness, overall QOL, emotional functioning, and constipation were found to be significantly associated with fatigue at baseline $(p<0.05)$. The logistic regression model showed that changes in hemoglobin and albumin levels, pain, dyspnea, physical functioning, and insomnia on QOL scale significantly contribute to the improvement in fatigue.

88. Effects of Community-Based Palliative Care on Health Care Utilization in Patients with Advanced Heart Failure

Temmy Latner Centre for Palliative Care, Mount Sinai Hospital Toronto, ON

Team/Contact Information:

Leah Steinberg, MD

Temmy Latner Centre for Palliative Care, Mount Sinai Hospital 60 Murray Street, 4th floor, Box 13

Toronto, Ontario, Canada

Lsteinberg@mtsinai.on.ca

Project Team Members:

Meghan White

Dr. Susanna Mak

Dr. Jennifer Arvanitis

Dr. Russell Goldman

Dr. Amna Husain

Topics: Palliative Care Delivery/Operations: Approaches to palliative care delivery for specific population types (e.g., heart failure/cancer/dementia/frailty)

Description:

In 2000, patients with advanced heart failure (HF) accounted for the second highest number of hospital days in Canada. We 
propose that providing home-based palliative care (PC) for these patients can meaningfully reduce the number of hospital visits and days in the hospital, with the ultimate goal of improving patient's overall satisfaction with care and quality of life.

In a quality improvement (QI) analysis of health care utilization of a sample of patients with advanced HF in a tertiary hospital in an urban setting, we measured and compared the rate of clinic visits, emergency department (ED) visits, hospital admissions, and days in the hospital before and after implementing home-based palliative care.

A total of 32 patients with HF (69\% female, $M=83.6$ years of age) were seen in the home-based PC program over a 2-year period. In a pre- and post-intervention comparison, meaningful reductions were seen in rates of clinic visits, ED visits, hospital admissions, and days in the hospital.

Home-based care of patients with HF can lead to decreased health care utilization. In turn, we hope it can reduce both patient and system burden and result in greater health-related quality of life for patients with advanced HF.

89. Developing a Protocol for Home Diuresis of Patients with Advanced Heart Failure

Temmy Latner Centre for Palliative Care, Mount Sinai Hospital Toronto, ON

Team/Contact Information:

Leah Steinberg, MD

Temmy Latner Centre for Palliative Care, Mount Sinai Hospital 60 Murray Street, 4th floor, Box 13

Toronto, Ontario, Canada

Lsteinberg@mtsinai.on.ca

Project Team Members:

Dr. Susanna Mak

Meghan White

Dr. Russell Goldman

Dr. Jennifer Arvanitis

Dr. Amna Husain

Topics: Palliative Care Delivery/Operations: Approaches to palliative care delivery for specific population types (e.g., heart failure/cancer/dementia/frailty)

\section{Description:}

Patients with advanced heart failure represent a population that is significant in number, health system utilization, and symptom burden. The condition is responsible for a high burden of hospitalization for symptoms that are associated with high mortality, and currently are difficult to manage in the ambulatory setting.

To address this patient and system burden we are developing a collaborative model of symptom management and homebased care for patients with advanced heart failure.

In collaboration with cardiology and primary care, our palliative care team is developing a program to facilitate homebased, multi-disciplinary care for patients with advanced heart failure. The program aims to develop education and process tools to advance home-based care of this population. These include a home diuresis protocol with guidelines for oral and intravenous diuretic therapy; an overview of how to assess and manage common symptoms in patients with advanced heart failure, and guidelines for rational withdrawal of cardiac medications.

Home-based care of patients with advanced heart failure that includes optimizing diuresis can lead to improved symptom management and a decreased number of hospitalizations. In turn, we hope it can reduce both patient and system burden and result in greater health-related quality of life for patients.
90. New and Revised Requirements for The Joint Commission Advanced Palliative Care Certification Program The Joint Commission

Columbus, $\mathrm{OH}$

Team/Contact Information:

Kathy K. Clark, MSN, RN

The Joint Commission

One Renaissance Boulevard

Oakbrook Terrace, IL 60181

(630) 792-5932

KClark@JointCommission.org

Project Team Members:

Tammy Croney, RN, DNP

Topics: This session will present TJC's Advanced Certification Program, including the new and revised standards, a review of the on-site survey process, and the value of obtaining and maintaining this certification for your organization's palliative care program.

Description:

During 2014, The Joint Commission reviewed its Advanced Certification in Palliative Care program, and researched changes in the field of palliative care that may necessitate modifications to the program. Using the 2013 Clinical Practice Guidelines for Quality Palliative Care (3rd edition), the National Hospice and Palliative Care Organization's Standards of Practice for Pediatric Palliative Care and Hospice (2010), and other recent literature, staff used a gap analysis to determine areas in the current program that needed revisions. These proposed revisions were written and then vetted by a panel of national palliative care experts. The main areas identified for modification included:

- Use of trained volunteers in a palliative care program

- Specific topics for staff orientation and ongoing education

- Types of assessments to be completed by interdisciplinary team members on admission to palliative care service, including quality of life, cultural, and spiritual assessment

- Requirement for the program to provide or make a referral for hospice care as needed

- Requirement that the program provide or refer family for bereavement services

- Specific pediatric requirements for programs that provide pediatric palliative care

The proposed new and revised requirements were also reviewed by the field via an on-line survey. After minimal revisions based on the survey results, the modifications to the program were approved by The Joint Commission's Board of Commissioners, and will go into effect July 1, 2015. The Certification review process was also revised to address the new and revised requirements in the program. We believe that these new and restructured requirements will be of value to currently certified palliative care programs, and that the modifications will encourage new organizations to pursue this respected advanced certification for their palliative care program.

The Joint Commission is currently developing palliative care performance measures, and plans to release them in mid-2016. We are currently doing research on community-based palliative care, to begin the work of expanding this certification program outside the hospital setting, and into community health care settings such as home care, hospice, and ambulatory health care organizations. 
91. Improving Do-Not-Resuscitate Discussions: A Framework for Physicians

The Ohio State University Wexner Medical Center

Columbus, $\mathrm{OH}$

Team/Contact Information:

Robert M. Taylor, MD

The Ohio State University Wexner Medical Center

246 Atwell Hall/453 W. 10th Avenue

Columbus, $\mathrm{OH} 43210$

(614) 293-2957

robert.taylor@osumc.edu

Project Team Members:

Jillian L. Gustin, MD

Sharla M. Wells-DiGregorio, PhD

Topics: Communication: Communication best practices (e.g., teaching goals of care conversations/advance care planning/family meetings)

Description:

Having DNR (Do-Not-Resuscitate) discussions with patients and their surrogates is a challenging task for most clinicians. To make such discussions more constructive, we provide a framework that stratifies patients into three general groups: (1) the basically healthy, (2) those with an advanced or chronic illness, and (3) the imminently dying. This framework permits physicians to tailor their discussions to the specific needs of each patient or surrogate and to make recommendations when appropriate. In discussing DNR orders, physicians struggle with two related challenges. The first is "How should they frame the discussion so that patients and surrogates understand the medical situation, including the benefits and burdens of their decisions?" The second is "To what extent is it appropriate to make recommendations?" The lack of "best practices" for communicating uncertainty further complicates DNR discussions. Although DNR orders should usually be discussed in the context of a broader conversation about the goals of care and advance care planning, they are sufficiently unique that it is instructive to consider them as a distinct issue.

92. Alternative Healing "Tools" for Caregivers

The Reiki Wind School \& Healing Center

San Diego, CA

Team/Contact Information:

Kathleen Cueva, BSN

The Reiki Wind School \& Healing Center

10524 Gretler Place

La Mesa, CA 91941

(619) 972-9895

kathy@reikiwind.com

Project Team Member:

Robert Bowen, RMT

Topics: Palliative Care Delivery/Operations: Innovative and emerging community-based palliative care models (e.g., home/ office/clinic/long-term care)

Description:

The "Healing Hands for Caregivers" program is designed for family caregivers, hospice and palliative care volunteers and staff members. These five simple "tools" are based on energy healing techniques and include comfort hand massage. They have been successfully used to promote relaxation, reduce pain and anxiety, and improve overall wellbeing for those who face physical, emotional, or mental challenges.
93. Building a Rural Ambulatory Palliative Care Program

The University of Texas Health Science Center at Tyler

Tyler, TX

Team/Contact Information:

Paula R. Brandon, MSN

The University of Texas Health Science Center at Tyler

11937 U.S. Hwy. 271

Tyler, TX 75708

(903) 877-8930

paula.brandon@uthct.edu u

Project Team Members:

Amy Newton, DO

Topics: Palliative Care Delivery/Operations: Innovative and emerging community-based palliative care models (e.g., home/ office/clinic/long-term care)

Description:

The University of Texas Health Science Center at Tyler is the sole academic medical center in the northeast Texas region providing ambulatory palliative care services to 28 underserved rural counties. This poster will demonstrate the barriers to building, program framework and guidelines, continuity of care, and challenges faced. The palliative care service is an adjunct specialty service that works collaboratively with other providers to educate and create an awareness related to appropriate referrals of patients who have life-limiting illnesses. The clinic is funded through the Texas 1115 Healthcare Transformation Waiver and expands patient service access. The program's framework is built upon a patient-centered multi-disciplinary team consisting of a registered nurse, social worker, chaplain, behaviorist, program specialist, and two palliative care board-certified physicians. The palliative care team provides patients with advance care planning, symptom assessment and management, psychosocial and spiritual support, counseling, and resource management. Challenges that the team faced include: foundational conceptualization to operational implementation, data capture, and template creation to meet electronic medical record (EMR) documentation requirements. The challenges resulted in recommendations related to information technology support, creation of EMR templates for each discipline, and the establishment of data collection methods prior to operationalization of the program.

94. Impact of Race on Pain Management Outcomes

UCSF Fresno Department of Hospice \& Palliative Medicine

Fresno, CA

Team/Contact Information:

Duc Minh Chung, MD

St. Joseph's Hospital and Medical Center

5573 North Brent Avenue

Fresno, CA 93723

(408) 824-0635

Dchung@fresno.ucsf.edu

Project Team Members:

Austin Sue, MD

James Simmons, DO

Christine Swift, MSN

Susan Hughes, MS

Tegest Hailu, MD

Patrick Macmillan, $M D$

Topics: Education: Targeting Education: Pain management and communication skills

Description:

Objective: Restrospective study to examine racial differences in pain outcomes following inpatient palliative care consultation. 
Participants were of African American, Asian/Others, Caucasian, and Latino origins.

Main Outcome: Pain score at various time periods: first 24hour period in hospital (including admission); 24 hours before the date/time of consultation; 24 to 48 hours after consult completion; and 24 hours before discharge. Pain scores via VAS scale.

Results: Most patients were Caucasian or Latino, at least 60 years old, diagnosed with cancer, with differences in length of stay and types of health insurance.

Median admission pain ranged from 5.3 to 6.9 , whereas median pain 24 hours prior to discharge ranged from 1.2 to 3.3. African Americans had the highest initial pain score of 6.9 and showed most reduction in pain after consultation and prior to discharge ( -4.5 and -4.9 , respectively). Asians had an initial pain score of 6; differences of -2.9 and -3.2 were observed after consultation and prior to discharge. Caucasians and Latinos had the same initial pain score of 5.3; however, greater pain reduction was seen in Latinos at discharge compared with Caucasians; -3.4 versus -2.0 ( $p$ values of 0.46 and 0.38 , respectively). There was an overall pain reduction in all groups combined, $p<0.01$.

\section{Palliative Care Specialists and Medicare Services}

UHC

Chicago, IL

Team/Contact Information:

Samuel Hohmann, PhD

UHC

155 North Wacker Drive

Chicago, IL 60606

(312) 775-4205

hohmann@uhc.edu

Project Team Members:

Marilyn Szekendi, PhD, RN

Erika Johnson, MHSA

Topics: Financing and Payer Collaborations: Financing/benefit and payment models

Description:

End-of-life health care continues to evolve as experiments with models of care yield new insights into best management practices. The emergence of palliative care medical specialists is the result of not only demand for these services, but also demonstration of the appropriateness of their integration into end-oflife care. Since 2009 palliative care specialists as providers of services to Medicare fee for service beneficiaries has grown dramatically. Based on Medicare fee for service claims data between 2009 and 2013, the number of palliative care providers grew from less than 50 to more than 480 . They provided care to fewer than 100 patients in 2009 and more than 3500 in 2013. The majority of care $(69 \%)$ was provided in hospitals. Office and outpatient settings accounted for another $20 \%$. About $46 \%$ of the care was provided in seven states (IL, MI, MA, CA, OH, NC, NY). Although other types of medical specialists provide palliative care counseling and care, palliative care medical specialists have been trained to manage end-of-life patients, and growth in training of palliative care medical specialists will need to track growth in the demand for these services.

\author{
96. Prevalence of Patients Appropriate for PC Referral \\ UHC \\ Chicago, IL \\ Team/Contact Information: \\ Marilyn Szekendi, $P h D, R N$ \\ $U H C$ \\ 155 North Wacker Drive
}

Chicago, IL 60606

(312) 775-4301

szekendi@uhc.edu

Project Team Members:

Jocelyn Vaughn, MA

Topics: Palliative Care Delivery/Operations: Successful approaches to conducting an effective needs assessment

\section{Description:}

Results of a point prevalence study conducted at 33 U.S. academic medical centers will be presented and will include a description of the size and characteristics of the population of inpatients appropriate for palliative care referral, along with the percentage of these patients who were referred to and/or received palliative care services. The results of a qualitative study exploring barriers and facilitators to palliative care referral will also be presented.

\section{Collaborative Team Activity Improves Outcomes}

United Regional Healthcare System

Wichita Falls, TX

Team/Contact Information:

Michelle Nelson, BSN

United Regional Healthcare System

1600 11th Street

Wichita Falls, TX

(940) 764-6714

mnelson@unitedregional.org

Topics: Palliative Care Delivery/Operations: Implementation of standardized interventions such as checklists and screening tools Description:

Palliative care project teams tested and implemented various screening and intervention tools to improve care coordination, communication, and care transitions.

The tools implemented included ESAS, PPS, and LACE.

We also implemented a screening process related to spiritual needs assessment and the subsequent documentation of the patients spiritual needs.

The primary outcomes of these tools provided direction on the symptoms we needed to most focus on: dyspnea and pain. Data indicate we have been improving our pain assessment and intervention process based on these findings.

Treatment preference discussions occur routinely and the challenges were documenting these discussions to ensure the preferences were passed on to the next care team. The patients who were DNR in the hospital setting are reviewed to appropriately obtain OOHDNR paperwork when applicable. This improved the transition to post-acute care settings.

The use of the LACE tool provided a score indicating a higher risk for readmission or mortality. The heart failure/ COPD patients with a score of 11 or greater on our service received a post-discharge phone call from the palliative care team/chronic care professional. Upon completion of discharge call, if a high risk for readmission is identified a referral is made to an APN for follow-up.

\footnotetext{
98. How Come They Don't Come? Identifying Reasons of Non-Show among a Palliative Care Outpatient Clinic University Health System, University of Texas Health Science Center at San Antonio, South Texas Veterans Healthcare System San Antonio, TX Team/Contact Information:

Sherry M. Williams, MD

University Health System, University of Texas Health Science Center at San Antonio
} 
South Texas Veterans Healthcare System

5903 Babcock Road, Apt. 903

San Antonio, TX 78240

(217) 620-1268

swilliams2@siumed.edu

Project Team Members:

Amune Blessing

Carmen Sanchez, MHA

Shuko Lee, MS

Jennifer Healy, DO

Sandra Sanchez-Reilly, MD

Topics: Palliative Care Delivery/Operations: Innovative and emerging community-based palliative care models (e.g., home/ office/clinic/long-term care)

Description:

Palliative care (PC) patients experience complex distress better addressed by an interdisciplinary PC outpatient clinic then by standard outpatient care. PC patients, like many patients, do not always keep appointments. These non-shows (NS) are missed appointments with no prior cancellation.

Objective: To assess factors associated with PC NS.

Methods: A telephone survey of patients/caregivers was performed of NS patients for appointments from November 2014 to April 2015 using a 19-question telephone script.

Results: $N=76$; Female $=39 ; 38$ agreed to participate, 21 declined, and 17 non-working phone numbers. Reasons identified for NS: 10 hospitalized, 7 deceased, 6 too sick, 3 hospice, 5 never seen in clinic/didn't know to attend, 4 no reminder call, 1 transportation, and 2 denied missing. Prior visits reported as beneficial by 13 of 14 patients. Benefits identified were: managing pain, symptoms, and spiritual concerns.

Conclusion: Patients with NS had many reasons for NS appointments such as hospitalization, symptom burden, and not knowing about appointment. Developing a more systematic appointment reminder system might free up appointments for other patients by removing deceased/hospitalized/hospice patients and making unaware patents better informed of appointments. Also close follow-up phone calls after NS to triaging symptoms/issues that lead to NS and improve patient care.

\section{Satisfaction Surveys: Timing of Inpatient Palliative} Care Consults

University of Arkansas for Medical Sciences Palliative Care Program

Little Rock, AR

Team/Contact Information:

Sarah E. Harrington, MD, FAAHPM

University of Arkansas for Medical Sciences Palliative Care Program

UAMS Slot \#508

4301 W. Markham

Little Rock, AR

(501) 686-8511

SEHarrington@uams.edu

Project Team Members:

Dianne Campbell, MSW

Topics: Other

Description:

Clinical practice guidelines suggest that patients and families participate in the evaluation of palliative care services. Additionally, feedback from referring physicians can be particularly helpful for engaging key stakeholders within a hospital as well for general quality improvement. In preparation for Joint Commission certification, we developed surveys for both sets of stakeholders.
Our team was particularly interested in timing of consultations within our system. Using the CAPC registry, we were able to compare our consult data with similar institutions. The survey for patients and families and the survey completed by referring physicians asked about timing of palliative care consults in order to gauge perception of timing. This poster will show the results of those targeted timing questions along with overall satisfaction rates. We will also present length of stay data (overall, admission to consult, and consult to discharge) to show where UAMS performs compared with similar institutions. We will discuss how we used these data for team development and quality improvement, as well as for Joint Commission certification. We will plan to make complete sample surveys available at the poster presentation.

100. A Palliative Nurse Practitioner Intervention to Improve Advance Care Planning and Supportive Care in Patients with Advanced Cancer

University of California, Los Angeles

Los Angeles,CA

Team/Contact Information:

Anne Walling, MD, PhD

Assistant Professor in Residence

University of California, Los Angeles

911 Broxton Avenue

Los Angeles, CA 90095

awalling@mednet.ucla.edu

Project Team Members:

Sarah D'Ambruoso

Sarah Hurvitz.

Robin Clarke

Andrew Hackbarth

Anne Coscarelli

Christopher Pietras

Jennifer Malin

Ann Zisser

Bruce Ferrell

Frances Watts

Anne Coscarelli

Thomas Strouse

Thomas Pier

Neil S. Wenger

Topics: Palliative Care Delivery/Operations: Innovative and emerging community-based palliative care models (e.g., home/ office/clinic/long-term care)

\section{Description:}

The simultaneous care model for palliative care provides high-quality care for patients with serious illness. Unfortunately, the U.S. health care system falls short of this goal, even in patients with advanced cancer. As the first phase of a system-wide advance care planning program, we used implementation science methods to test if a nurse practitioner (NP) with structured palliative care training using evidenced-based principles of communication and a model that cultivates prognostic awareness with palliative care physician oversight can improve the quality of care provided to patients with advanced cancer in an academic health system. The intervention was evaluated using electronic health record data to compare rates of advance care planning and hospice referral for patients with advanced cancer seen by two target oncologists compared with all other advance cancer patients in clinics without NP intervention. There were no differences in these measures at baseline, but after one year of the program, data suggest that this model of palliative care delivery increased rates of advance care planning among advanced cancer patients (30\% versus $17 \%$, 
$p<0.05)$ and hospice enrollment among decedents (53\% versus $23 \%$ ). Improved payment models are needed to help sustain and grow programs of outpatient palliative care delivery.

101. The Tipping Point Demands a Return to Primary Palliative Care: System- and Continuum-Wide Education Using the End-of-Life Nursing Education Consortium Curriculum University of Kansas Hospital

Kansas City, KS

Team/Contact Information:

Karin B. Porter-Williamson, MD

University of Kansas Hospital

6040 Delp

Mailstop 1020

3901 Rainbow Boulevard

Kansas City, KS 66160

(913) 588-3807

kporter-williamson@kumc.edu

Project Team Members:

L. Redford

M. Fox

Topics: Education: Methods of training in primary/generalist palliative care

\section{Description:}

As palliative care programs mature, the need to empower frontline providers to address primary palliative care needs increases. For institutions in rural states with a broad geographic referral base, an even wider net for education is necessary. Nurses are an important vector to spread this knowledge within their teams; however, they cite lack of competency in this area. The ELNEC (End-of-life Nursing Education Consortium) curriculum provides structured content to enhance nurses' competency to provide palliative care.

Intervention: A multi-disciplinary group of five individuals from the University of Kansas Hospital attended a Train-thetrainer ELNEC Summit in January 2012, then started ELNEC education for internal and community providers across the state of Kansas via ITV. Dissemination includes training 76 staff nurses, chaplains, and social workers from hospital and ambulatory sites as Palliative Care Champions. Consequently, these champions have identified service gaps in their practice areas and implemented process improvement strategies addressing the needs. Champions also help teach a bi-annual 1-day ELNEC program. From this, 280 additional providers from our institution and across Kansas have been trained. Participants show improved knowledge and confidence in addressing palliative needs. The ELNEC curriculum is evidence-based and effective to improve systems' delivery of primary palliative care.

\section{Characterizing the Role of Pharmacists in Palliative Care}

University of Maryland School of Pharmacy

Baltimore, MD

Team/Contact Information:

Kathryn Walker, PHARMD

University of Maryland School of Pharmacy

201 E. University Pkwy.

33rd Street Building, Suite 415

Baltimore, MD 21218

(410) 554-2923

dstewart@rx.umaryland.edu

Project Team Members:

Diana Stewart, PharmD

Mary Lynn McPherson, PharmD
Topics: Palliative Care Teams: Staffing the interdisciplinary team including staffing benchmarks and maximizing the roles of pharmacists/social workers/chaplains/nursing and medicine Description:

A crucial component of palliative care is the provision of services to a patient by an interdisciplinary team. This team is often comprised of a physician, a registered nurse, a social worker, and a chaplain. Pharmacists make valuable contributions to team-based patient care by recommending appropriate therapy selections, providing medication education to patients and care providers, monitoring for side effects and therapeutic response, and assisting with the development of medicationbased policies. The role of pharmacists as part of the interdisciplinary teams, however, is poorly defined. The purpose of this research is to characterize the presence of pharmacists in palliative care teams, their role and responsibilities, and training in palliative care.

\section{PACCT-Building Bridges-One Way to Grow a Program}

University of Minnesota Masonic Children's Hospital/Fairview Home Care and Hospice

Minneapolis, MN

Team/Contact Information:

Naomi Goloff, MD

University of Minnesota Masonic Children's Hospital/Fairview Home Care and Hospice

Minneapolis, MN

ngoloff1@fairview.org

Project Team Members:

Jody Chrastek, RN, DNP

Topics: Palliative Care Delivery/Operations: Innovative pediatric palliative care programs including combined adult/pediatric models

\section{Description:}

Pediatric palliative care (PPC) provision in the hospital and community has grown rapidly in the last decade. However, in the rapidly evolving health care arena, services can change quickly. This poster shares the story of how a change in community services for PPC challenged a large health care system to collaborate in a new way to provide care. A partnership was formed between a children's hospital, an adult home care, and an adult hospice program to create a Pediatric Advanced Complex Care Team (PACCT).

The goal was to develop a sustainable program to provide both inpatient and home-based interdisciplinary care for children with life-threatening conditions who needed complex medical care, palliative care, or hospice. Marketing, education, collaboration, culture change, and support from leadership of all entities were needed to make this happen.

Margaret Wheatley's theory of change (www.margaretwheatley. com/articles/emergence.html) was a helpful framework as the program developed. This poster will show the time line, strategies, educational methods, resources used, and lessons learned.

104. Development of a Family Meeting Assessment Tool University of Texas Health Science Center at San Antonio San Antonio, TX

Team/Contact Information:

Yuya Hagiwara, $M D$

University of Texas Health Science Center at San Antonio 7703 Floyd Curl Drive MSC 7875

San Antonio, TX 78229

hagiwara@uthscsa.edu 
Topics: Education: Targeting Education: Pain management and communication skills

\section{Description:}

Learning how to lead family meetings is an important skill for physicians, nurses, and others who care for patients with serious illnesses and their families. There is limited evidence exploring methods to assess the skill to perform family meetings. Our goal was to develop and validate an observational tool to assess trainees' ability to perform and lead family meetings. Building on evidence from literature search, the Family Meeting Objective Structured Clinical Examination Assessment Tool (FMOSCEAT) was developed by our expert panel to evaluate best practice behaviors when leading a family meeting. As part of our palliative care curriculum, 4th-year medical students completed a Family Meeting OSCE, where each student assumed the physician role leading a complex family meeting with two standardized family members. Two interdisciplinary evaluators were paired to observe and rate randomly chosen students' performances in the OSCE using the FMOSCEAT. Inter-rater reliability was measured using percent agreement. Internal consistency was measured using Cronbach's alpha. One hundred thirty-three trainees and 26 interdisciplinary evaluators participated. Overall rater agreement was $84 \%$ and Cronbach's alpha was 0.85 . Evidence- and expert-based content, high interrater reliability, and good internal consistency provided initial evidence of validity for this novel assessment tool.

105. How Come They Don't Show Up?: Assessment of NoShows in Outpatient Palliative Care

University of Texas Health Science Center San Antonio

University Health System

South Texas Veterans Health Care System

San Antonio, TX

Team/Contact Information:

Jennifer M. Healy, DO

University of Texas Health Science Center San Antonio

University Health System

South Texas Veterans Health Care System

5652 Lockhill Road

San Antonio, TX 78240

(210) 870-8180

Healyj@uthscsa.edu

Project Team Members:

Kathryn Sawey, DO

Sherry Williams, MD

Jennifer Healy, DO

Shuko Lee, $M S$

Sandra Sanchez-Reilly, MD

Topics: Other

Description:

Background: Palliative care clinics (PCC) are designed to meet the needs of seriously ill patients. PCC help patients and families deal with uncontrolled symptoms and establish goals of care to avoid crisis situations (unnecessary hospital admissions).

Methods: Retrospective chart review on initial/established patients with no-show appointments (NS) from 2013 to 2015. Examined variables included functional performance, symptom burden, and pain scores.

Results: $N=100$ patients seen in PCC. Appointments were scheduled within 3 days of inpatient discharge. Sixty-one percent were NS, $39 \%$ had PCC appointment prior to NS; $7 \%=$ admitted to emergency room (ER) or hospital on day of NS and $9 \%=$ had ER visit within 2 weeks of NS; $21 \%$ of
$\mathrm{NS}=$ had further NS. There was non-significant worsening trend for all symptoms/pain scores after NS. Palliative Performance Scores showed significant decline in functional status after NS with median score decrease from $60 \%$ to $40 \%$ $(p=0.0373)$. There was a significant decrease in spiritual/ existential suffering $(p=0.0001)$ and social issues/suffering $(p=0.0166)$ even with NS.

Conclusion: Patients with NS faced barriers such as uncontrolled symptoms and ER/hospital admissions. NS correlated with a decline in functional status. Patients had decrease in spiritual/social suffering, which may be a reflection of the high level of support PC offers.

\section{Complex Lives, Comprehensive Services}

University of Texas Health Science Center San Antonio

University Health System

South Texas Veterans Health Care System

San Antonio, TX

Team/Contact Information:

Jennifer M. Healy, DO

University of Texas Health Science Center San Antonio

University Health System

South Texas Veterans Health Care System

5652 Lockhill Road

San Antonio, TX 78240

(210) 870-8180

Healyj@uthscsa.edu

Project Team Members:

Rachel Vandermeer, MD

Glen Medellin, $M D$

Jennifer Healy, DO

Sandra Sanchez-Reilly, MD

Topics: Palliative Care Delivery/Operations: Innovative pediatric palliative care programs including combined adult/pediatric models

\section{Description:}

Background: Pediatric palliative services nationwide enroll children with life-limiting, but not acutely life-threatening chronic illness. Children receiving inpatient palliative services, the most prevalent service delivery model, have a one-year survival rate of nearly $75 \%$. In response to the long survival rate and the need for outpatient palliative services, University Health System (UHS) developed a medical home clinic that provides integrated palliative and primary care for children with medical complexity or incurable illness.

Methods: A retrospective chart review was performed for all patients seen in the UHS Comprehensive Care Clinic (UHSCCC) between September 2013 and February 2015.

Results: In a 1.5-year period, the UHS-CCC performed 2338 visits for 359 children, average $=4.3$ visits/patient $/$ year. Sixty-six percent $=$ had gastrostomy for nutrition; $25 \%=$ had tracheostomy; $30.3 \%=$ required ventilator support; $56.9 \%=$ received home health nursing. The death rate $=4.3$ deaths $/ 100$ patients/year; $4.8 \%=$ enrolled in concurrent hospice at some point over the study period. The breakdown of diagnoses was: $41.5 \%=$ neuromuscular, $33.5 \%=$ congenital, $5.3 \%=$ oncologic, $1.6 \%=$ respiratory, $5.9 \%=$ cardiovascular, $4.3 \%=$ gastrointestinal, and $8 \%=$ other.

Conclusion: This demographic study describes the high complexity of outpatients receiving pediatric palliative care in UHS-CCC. The outpatient pediatric palliative population is dependent on both technology and community professionals. The death rate requires supporting patients at end of life. 
107. Palliating Interns: The Impact of a Palliative Care Rotation on Internal Medicine Interns

University of Texas Health Science Center San Antonio

University Health System

South Texas Veterans Health Care System

San Antonio, TX

Team/Contact Information:

Jennifer M. Healy, DO

University of Texas Health Science Center San Antonio

University Health System

South Texas Veterans Health Care System

5652 Lockhill Road

San Antonio, TX 78240

(210) 870-8180

Healyj@uthscsa.edu

Project Team Members:

Shuko Lee

Sandra Sanchez-Reilly, MD

Topics: Education: Methods of training in primary/generalist palliative care

Description:

Background: Many studies look at efficacy for including palliative care (PC) for medical students, yet incorporation of PCrotations for residents has not been required nor well studied. Core topics for PC include communication, symptom management, and working with inter-professionals. These may be taught in didactics, but supervised practice has the greatest potential for impact.

Methods: Forty-two medicine interns (26 categorical; 15 preliminary); $50 \%$ categorical completed PC rotation (13) and $100 \%$ completed PC-focused didactics (42). Upon completion of internship, web-based surveys were sent that included pre- and postreflection. Survey included validated Self-Efficacy in PC Scale (assesses communication efficacy, patient management, multiprofessional teamwork) and the Thanatophobia Scale (assesses PC attitudes). McNemar's test was used for statistical analysis.

Results: Completed survey $=10$ (8 categorical; 2 preliminary), female $=20 \%$. Caucasian $=40 \%$, Hispanic $=50 \%$, and Pacific Islander $=10 \%$; age $=20$ to 39 . Completed $\mathrm{PC}$ rotation $=5$. Overall, pre-year attitudes were positive and remained positive; $40 \%$ of the 5 interns with negative attitudes in caring for PC patients shifted to a positive attitude $(p=0.0047)$. There was statistically significant improvement in self-efficacy in all areas-most notable being interns' ability to assess patient needs and prescribe appropriate pain medications. When comparing those who completed PC rotation against no PC rotation, there was higher percent of shifting to confident in completers ( $p=$ non-significant due to small number).

Conclusion: This study suggests that IM interns greatly benefit from a PC clinical experience.

\section{Benefits of Palliative Care Consultation in a Medical} ICU

University of Texas Health Science Center San Antonio

University Health System

South Texas Veterans Health Care System

San Antonio, TX

Team/Contact Information:

Jennifer M. Healy, DO

University of Texas Health Science Center San Antonio

University Health System

South Texas Veterans Health Care System

5652 Lockhill Road

San Antonio, TX 78240

(210) 870-8180

Healyj@uthscsa.edu
Project Team Members:

Bethany Kapp, $M D$

Jennifer Healy, DO

Shuko Lee, MS

Sandra Sanchez-Reilly, MD

Topics: Palliative Care Delivery/Operations: Approaches to palliative care delivery in specific hospital settings (e.g., ICU, ED)

Description:

Background: It has already been documented that inpatient palliative care consultation (PCC) can reduce length of stay (LOS) and costs among seriously ill individuals admitted to medical intensive care units (MICU). More information on MICU-PCC cost impact may contribute to additional administration support and more resources to care for terminally ill patients.

Methods: We analyzed de-identified data for a 40-month period (January 2012 to April 2015), including all patients admitted to MICU at our academic county hospital. Of the 86,131 admissions, there were 1449 patients with 3829 visits to MICU. We matched PC patients with standard care (SC) patients using DRG and discarded those without $3+$ matches. $N=1347$ matched MICU patients; 40\%= female; 931/1347= with PCC.

Results: There was a statistically significant decrease in PPC group with median LOS of 9 versus 15 days in the non-PCC group $(p<0.0001)$. Difference in mean total charges was $\$ 79,348$ with non-PCC group at $\$ 196,293$ versus PCC group at $\$ 116,945$ ( $p<0.0001)$. There was non-significant trend for decreased repeat MICU admissions with PCC group mean $=1.05$ and non-PCC group mean $=1.08(p=0.089)$.

Conclusion: MICU-PCC significantly decreased both LOS and costs. Although not significant, there was down trend in repeat MICU admissions with MICU-PCC.

109. Nurses Have the Magic Touch: Determining the Effect of Embedding a Palliative Care Nurse within the MICU University of Texas Health Science Center San Antonio University Health System

South Texas Veterans Health Care System

San Antonio, TX

Team/Contact Information:

Jennifer M. Healy, DO

University of Texas Health Science Center San Antonio

University Health System

South Texas Veterans Health Care System

5652 Lockhill Road

San Antonio, TX 78240

(210) 870-8180

Healyj@uthscsa.edu

Project Team Members:

Christina Mireles, $M D$

Bethany Kapp, MD

Jennifer Healy, DO

Shuko Lee, MS

Sandra Sanchez-Reilly, MDS

Topics: Palliative Care Delivery/Operations: Approaches to palliative care delivery in specific hospital settings (e.g., ICU, ED)

Description:

Background: Critically ill medical intensive care unit (MICU) patients are at high risk for significant morbidity and mortality. As such, goals of care and end-of-life decisions are warranted. Prior studies evaluated palliative care (PC) consultants role within the MICU; however, embedding PC nurse practitioners (NP) in the MICU and its effects on length of stay (LOS) and overall admission costs (OAC) has not been assessed. 
Methods: Forty-month period (2012 to 2015) de-identified data were analyzed, including all patients admitted to MICU. Of the 86,131 hospital admissions, there were 1449 patients with 3829 MICU visits. We matched PC patients with standard care (SC) patients using DRGs. $N=1347$ matched; 40\% female; 931/ 1347 patients had PC-consult. Repeat MICU admissions, LOS, and OAC were compared for PCC and no-PPC groups before the embedded PC NP and after.

Results: There was a significant decrease in LOS in PCC arm after embedding PC NP (from 16.9 to $12.2 ; p=0.0006$ ). There was non-significant decrease in LOS in the non-PCC arm (from 24.3 to $22.5 ; p=0.8680$ ) and in OAC: PCC-arm (from $\$ 129,014$ to $\$ 109,807 ; p=0.1539)$. Interestingly, there was a nonsignificant increase in OAC for non-PCC arm (from $\$ 173,452$ to $\$ 204,599 ; p=0.0664)$.

Conclusions: This study revealed significant LOS decrease upon embedding PC NP within the MICU. However, there was non-significant difference in OAC.

\section{A Brief End-of-Life Communication Curriculum for} Internal Medicine Interns

University of Texas Health Science Center San Antonio

University Health System

GEC/GRECC South Texas Veterans Health Care System

San Antonio, TX

Team/Contact Information:

Katie Stowers, DO

University of Texas Health Science Center San Antonio

University Health System

GEC/GRECC South Texas Veterans Health Care System

7703 Floyd Curl Drive

San Antonio, TX 78231

(210) 358-0594

katiehstowers@gmail.com

Project Team Members:

Sandra Sanchez-Reilly

Topics: Communication: Communication best practices (e.g., teaching goals of care conversations/advance care planning/ family meetings)

\section{Description:}

Internal medicine interns are the physicians spending the most time with hospitalized patients, frequently assisting with end-of-life (EOL) decision making. However, most report feeling ill-equipped for these situations. We sought to assess a brief hands-on EOL communication curriculum for Interns (PC-I). Twenty-four participated in PC-I comprised of 1-hour didactic, 1-hour role-play, and two observed structured clinical encounters: Breaking Bad News (BBN), Family Meeting (FM) with real-time evaluation and feedback. Pre/post curriculum self-assessment of competence was completed. Preceptor evaluation/feedback identified improvement themes: 1) setting meeting agenda "warning-shot"; 2) avoiding medical jargon; 3) discussing prognosis; 4) appropriate utilization of surrogate decision-maker. Intern impressions identified four major themes: 1) safe environment for skills practice; 2) confidence performing skills in clinical setting; 3) valued feedback; and 4) patients/families appreciate direct conversations about death/ dying and prognosis. Interns showed improvement in confidence discussing patient's death with patient $(p<0.05)$, with family $(p=0.07)$, and with family during bereavement $(p<0.05)$. A brief, hands-on, multi-modality EOL communication curriculum is a valuable learning experience for interns at early stages of their career, which can improve confidence and identify specific areas for continued skill development.
Further research is needed to assess long-term impact as it pertains to improved clinical outcomes.

111. Protecting Our Vulnerable Population: Screening for Mistreatment in Palliative Care

University of Texas Health Science Center San Antonio

University Health System

GEC/GRECC South Texas Veterans Health Care System

San Antonio, TX

Team/Contact Information:

Katie Stowers, DO

University of Texas Health Science Center San Antonio

University Health System

GEC/GRECC South Texas Veterans Health Care System

7703 Floyd Curl Drive

San Antonio, TX 78231

(210) 358-0594

katiehstowers@gmail.com

Project Team Members:

Heather Veeder

James Linden

Shuko Lee

Sandra Sanchez-Reilly

Topics: Palliative Care Delivery/Operations: Implementation of standardized interventions such as checklists and screening tools Description:

Palliative care (PC) patients are a vulnerable population. Although the incidence of mistreatment in other vulnerable populations has been defined, approaching $10 \%$ of the elderly, the incidence in PC has not. We sought to assess the rate of PC patients who establish concern for mistreatment (ECM) and identify risk factors by screening our PC patients for mistreatment using two mistreatment screening tools validated among the elderly: Elder Abuse Suspicion Index (EASI) and Caregiver Abuse Screen (CASE). Sixty-four patients were screened over 30 days; 11 (17.19\%) ECM. A significant relationship was found between PC diagnoses and ECM, $(p=0.01)$. EASI question \#2 (preventing access to resources/people/medical care, $p=0.02$ ), \#3 (verbal shaming, $p<0.001$ ) and CASE question \#2 (caregiver shameful of actions, $p=0.005$ ), \#4 (caregiver acting rough, $p=0.02$ ), and \#7 (caregiver exhaustion, $p=0.006$ ) were significant to ECM. The rate of PC patients who ECM is similar to other vulnerable populations. ECM risk factors included PC diagnoses; further risk factor identification was limited by small sample size and variable applicability of elderly-targeted validated scales. Larger scale studies are needed to identify risk factors for PC mistreatment, and to develop validated PC mistreatment screening tools, ultimately improving patient care for vulnerable PC patients and targeting risk factors.

\section{Implementation of Home-Based Palliative Care with CRNPs}

University of Pittsburgh Medical Center Palliative and Supportive Institute

Pittsburgh, PA

Team/Contact Information:

Denise Stahl, MSN

Executive Director

University of Pittsburgh Medical Center Palliative and Supportive Institute

113 Kilarney Drive

Hunker, PA 15639

(412) 864-2856

stahld@upmc.edu 
Topics: Palliative Care Delivery/Operations: Innovative and emerging community-based palliative care models (e.g., home/ office/clinic/long-term care)

\section{Description:}

As an Integrated Delivery and Financing System, the University of Pittsburgh Medical Center (UPMC) and the UPMC Health Plan identified gaps in services related to care transitions from facility to home. These gaps included breakdown in communication across the health care team, limited scope of traditional home health services, and inconsistent follow-up for patients with serious chronic illness. A collaborative effort between the UPMC Palliative and Supportive Institute (PSI), the health plan, and the systemowned home health agency was developed. Specifically, PSI established an independent practice of specialty-trained certified registered nurse practitioners to support two new payor/provider models of care in community. With autonomy of practice and customized documentation tools, the CRNP visits patients in the home, collaborates directly with the patient's primary care physician to minimize the burden of serious chronic illness, establish a value-based plan of care, and prevents unnecessary emergency room visits or re-hospitalization. A "Home Transitions" program leverages resources available within the home health team and enhances services with the CRNP, a pharmacist, and medical directors. The "Advanced Illness Care" program is designed to support patients with life-limiting illness/poor prognosis and is provided by a CRNP and a team of specialty-trained clinical social workers.

113. Increasing Palliative Medicine Utilization in the Surgical Intensive Care Unit at An Urban, Safety-Net Hospital UT Southwestern Medical Center/Parkland Hospital

Dallas, TX

Team/Contact Information:

Brian H. Williams, MD

UT Southwestern Medical Center/Parkland Hospital

5323 Harry Hines Blvd. E5.508

Dallas, TX 75390-9158

(214) 648-3343

brian.williams@utsouthwestern.edu

Project Team Members:

Brian H. Williams, MD, FACS

Nadine Semer, MD

Elizabeth Paulk, MD

Ernestina Damas, BSN, RN

Jessica Jegstrup, BSN, RN

Natalie Provenzale, $M P H, R N$

Deborah Boyer BS, RN

Topics: Palliative Care Delivery/Operations: Approaches to palliative care delivery in specific hospital settings (e.g., ICU, ED)

\section{Description:}

The SICU in our urban, university-affiliated, county hospital is a closed, 28-bed unit with over 1200 admissions each year. We hypothesized that there was an unmet need for palliative medicine input with complex SICU patients and completed a process improvement review that confirmed palliative medicine referral was rarely considered. In an effort to increase palliative medicine utilization, we implemented a SICU screening protocol done daily by the bedside critical care registered nurse (CCRN). The goal was to identify patients appropriate for palliative medicine consultation by establishing objective "trigger" criteria incorporated into the daily nursing assessment. The selected criteria were based on factors identified by members of the American College of Surgeons Surgical Palliative Care Task
Force, and adapted by an interdisciplinary team consisting of palliative medicine specialists, surgical critical care specialists, and SICU nurses. Our pilot study demonstrated an increase in palliative medicine referrals. We also demonstrated many appropriate patients still were not identified for referral. Our future plans are to prospectively track the increase in referral volume, evaluate the impact on palliative medicine service clinical capacity, and develop a formalized curriculum to train SICU nurses in primary palliative care.

114. Honoring Fallen Veterans with Memorial Procession VA WNY Healthcare System

Buffalo, NY

Team/Contact Information:

Jaclyn Schneider, MD

VA WNY Healthcare System

3495 Bailey Avenue

Buffalo, NY 14215

(716) 862-8994

Jaclyn.schneider@va.gov

Project Team Members:

Kirti Joseph, $M D$

Deborah Medakovich, RN, MSN

Nancy Dux, LMSW

Amy Case, $M D$

Topics: Palliative Care Delivery/Operations: Approaches to palliative care delivery in specific hospital settings (e.g., ICU, ED)

\section{Description:}

The VA WNY Healthcare Center has implemented into the standard operating procedures a memorial procession for all veterans who die in the hospital. This has increased staff selfawareness of the specialized needs of the dying veteran and family. Should a family member(s) wish to have a memorial procession, the nurse informs the staff and obtains a memorial procession pack which includes a morgue cart that resembles a coffin with an American flag. Nurses who have the prepared the body escort the deceased to where the family and staff are gathered. All staff is asked to gather on either side of the room or corridor. The POC thanks them for the privilege and honor of taking care of their loved one. The POC relates any personal anecdotes or stories, asks family or staff if anyone has anything else to share. The POC then asks permission to read a poem called "Remembered Joy" or a poem/story of one's own choosing. POC asks family if they are ready for "the warrior's final walk." The nurses lead the procession by pushing the morgue cart slowly with the family following behind while taps is being played and other veterans in the hallway salute.

\section{Redesigning the Role of the Palliative Care Team to Expand Services}

Vidant Health

Greenville, NC

Team/Contact Information:

Janet P. Moye, PhD, RN

Vidant Health

2100 Stantonsburg Road

Greenville, NC 27834

252-847-1242

janet.moye@vidanthealth.com

Project Team Members:

Tae Joon Lee, MD

Topics: Palliative Care Delivery/Operations: Innovative hospital-based palliative care models 


\section{Description:}

Our 900-bed hospital is the flagship hospital for the system's nine hospitals and is located in a largely rural area in eastern North Carolina. The 18-bed Palliative Care Unit (PCU) has existed for years but has been underutilized.

The primary reasons for the underutilization included lack of patient and provider knowledge of palliative care and the remote location of the PCU in the hospital away from the intensive care and intermediate units where most of the provider's patients are located creating efficiency challenges.

To bridge this gap, a new care model was implemented and the palliative care provider team (PCPT) was expanded to incorporate an attending role in addition to the traditional consultant role. In this model, admitting providers have the option of continuing to care to their patient after transfer to PCU or transferring care to the PCPT.

This innovation has increased knowledge of palliative care, promoted collaboration, and has improved continuity of care. Additionally, it has resulted in better utilization of the PCU for palliative-appropriate patients and increased provider, patient, and family satisfaction. Metrics include percentage of expected hospital deaths in the PCU compared with intensive care units, utilization of the PCU, and patient, family, and provider satisfaction data.

116. Improving Outcomes through Palliative Care-ICU Collaboration

Vidant Health

Greenville, NC

Team/Contact Information:

Janet P. Moye, PhD, RN

Vidant Health

2100 Stantonsburg Road

Greenville, NC 27834

252-847-1242

janet.moye@vidanthealth.com

Project Team Members:

Tae Joon Lee, MD

Topics: Palliative Care Delivery/Operations: Approaches to palliative care delivery in specific hospital settings (e.g., ICU, ED)

Description:

Recognizing the growing and unmet needs for palliative care in our 900-bed hospital, our leadership supported expansion and enhancement of the palliative care team. To bridge this gap, a medical director was hired to manage all of the hospice and palliative care activities, and the nurse practitioner staff was doubled.

In aligning activities with the organization's mission and strategic goals, the first priority was to facilitate "right care, right place, right time, right cost."

Reviews of deaths within the organization revealed that a greater than expected number of patients died in the intensive care units. The team focused on being accessible to providers and clinical staff in the intensive care units and developed partnerships to provide more timely education and consultation for patients and families. Palliative care consult availability was increased to 7 days a week from 5, ready access to the consultant was enhanced, and time from order to completion of consult was dramatically shortened.

As a result, the number of palliative care consult requests increased significantly and earlier palliative interventions occurred. Other outcomes include decreased length of stay for patients who die in the hospital and increased number of deaths in the Palliative Care Unit compared with ICU deaths.

\section{VNS/Hospital Partnership for In-Home Palliative}

Care

Visiting Nurse Service of Northeastern New York

Schenectady, NY

Team/Contact Information:

William Vacca, MD

Visiting Nurse Service of Northeastern New York

Schenectady, NY

wvacca@nycap.rr.com

Project Team Members:

Carol S. Weisse, PhD (Union College)

Katherine Pouliot (Union College)

David S. Pratt, MD, MPH (Visiting Nurse Service of Northeastern NY)

Phil DiSorbo, MA (Ellis Medicine)

Bill Vacca, MD (Care Choices)

Topics: Palliative Care Delivery/Operations: Innovative and emerging community-based palliative care models (e.g., home/ office/clinic/long-term care)

\section{Description:}

There is a growing need for home-based palliative care services, especially for seriously ill individuals who want to avoid hospitalizations and remain with their regular outside care providers. This poster will describe Care Choices, a new inhome palliative care program provided through a visiting nurse service (VNS) and community hospital serving New York's Capital District. The program was evaluated through a service learning partnership with researchers at a local college who collected data on patient satisfaction, quality of life, and hospitalizations among enrollees over the course of 1 year. Phone interviews were conducted on 123 patients at baseline, 1 month, and 3 months on service. Results revealed that patients were highly satisfied with their initial care and reported greater satisfaction and stable symptom management over time. In addition, fewer emergency room and inpatient hospital admissions occurred while on the service. The results suggest that an inhome palliative care program offered jointly through a local VNS and community hospital may be a successful model for providing the full spectrum of palliative care services in a patient's home, thereby satisfying seriously ill patients' desires to remain at home, manage symptoms, retain regular care providers, and reduce hospitalizations.

118. End-of-Life Care for Dementia Patients in the Program of All-inclusive Care for the Elderly (PACE): A Quality of Care Assessment

Wake Forest University

Winston Salem, NC

Team/Contact Information:

Jennifer Gabbard, MD

Assistant Professor

Wake Forest University

2336 Hartfield Circle

Winston Salem, NC 27103

jgabbard@wakehealth.edu

Project Team Members:

Matthew McNabney

Topics: Other

Description:

Background: The majority of Americans want to die at home, but this is particularly challenging for patients with dementia. The mission of PACE is to allow patients to remain at home until death. The goal of this project was to assess quality 
measures during the last 6 months of life for patients with the diagnosis of dementia while enrolled in PACE.

Methods: Retrospective study starting at 6 months prior to death for patients on PACE with dementia.

Results: Seventy charts were reviewed; $78 \%$ of patients had advance directives but only $38 \%$ of patients had documentation about preferences regarding hospitalization. None had documentation that spiritual, existential, or bereavement concerns were addressed. Forty-six percent of patients were not screened for pain, $50 \%$ were not screened for shortness of breath, and of those who were only $8 \%$ were on morphine or any additional medication beyond bronchodilators and oxygen. The average rate of admissions in the last 6 months of life was only 0.9 with only $7 \%$ of patients dying in the hospital.

Conclusion: This PACE program allowed most patients with dementia to die outside of a hospital. However, there needs to be more standardization in quality metrics to ensure high quality of life for dementia patients while dying on PACE.

119. The WellMed Bridges in Complex Care Program: Comprehensive Palliative Care across the Continuum

WellMed Medical Management

San Antonio, TX

Team/Contact Information:

Elizabeth J. Glazier, MD

Medical Director, Palliative Care Program

WellMed Medical Management

1719 Fawn Bluff

San Antonio, TX 78248

(205) 639-8805

eglazier@wellmed.net

Project Team Members:

Dr. Wesam Aziz

Dr. Leigh Fredholm

Dr. Paul Garcia

Dr. Tia Hooper

Dr. R. Martinez

Dr. Liliana Oakes

Dr. Ron Stephens

Dr. Nancy Torres

Dr. Deidra Woods

Amy Quintero, NP

Topics: Palliative Care Delivery/Operations: Innovative and emerging community-based palliative care models (e.g., home/ office/clinic/long-term care)

Description:

The WellMed's "Bridges" community-based palliative care program launched in San Antonio in August 2013. The palliative care team, which consists of RNs, LVNs, MAs, SWs, NPs, and MDs, utilize a multi-disciplinary approach to patient care. Working alongside the patient's primary care physician, we are able to provide patient-centered care, often in the comfort of the home. Bridges is provided at no cost to WellMed members diagnosed with chronic, life-limiting conditions. The initial evaluation includes geriatric and palliative care assessments either in the patient's home or in one of our subspecialty palliative care clinics. The program focuses on effective symptom management, care coordination, and comprehensive advance care planning. In San Antonio, the program has demonstrated a greater than $40 \%$ cost reduction through the avoidance of unnecessary hospitalizations and emergency room visits. Transitions to hospice occur earlier, which has allowed $90 \%$ of our patient deaths to occur in the home. Due to the success of the program, WellMed has expanded Bridges into El Paso, Austin, Corpus Christi, and the Rio Grande Valley. By targeting and enabling palliative services for patients much earlier in the illness trajectory than hospice, the Bridges program is "filling the gap" between serious illness and end-of-life care.

\section{Rothman Index as a Palliative Consultation Trigger}

Yale New Haven Hospital

New Haven, CT

Team/Contact Information:

Rebecca Gagne Henderson, APRN, ACHPN, PhD(c)

Yale New Haven Hospital

1450 Chapel Street

New Haven, CT 06511

(203) 867-8262

rebecca.gagnehenderson@ynhh.org

Project Team Members:

Joan Rimar, RN, DNSc

Stephen Allegretto, BS, $M P H$

Richard Hintz

Topics: Palliative Care Delivery/Operations: Implementation of standardized interventions such as checklists and screening tools

\section{Description:}

The Rothman Index (RI) is a peer reviewed and validated formula that provides a real-time, disease agnostic continuous measure of patient condition based on existing data sitting in the electronic medical record. The RI is fully integrated with our EMR system, and is used as an early warning system for unexpected deterioration. The RI is available and integrated in many of the most widely used EMRs.

The Palliative Care Service Manager (PCSM) reviewed the charts of 112 patients with an RI $<40$ and a LOS $>5$ days in the hospital during the month of February 2014. The PCSM applied a palliative screening to those charts. Ninety-five percent of the patients screened with RI 40 and a LOS $>5$ days met criteria for palliative care. The PCSM informally educated physicians regarding the significance of the low RI scores. Over the 3-month pilot, the manager's caseload increased from 9 to 20 patients per month in the pre-pilot period, to 31 by the pilot's third month. The disposition of such patients showed a decrease of hospital mortality and increased access to palliative care. This small pilot has implications for future quality improvement efforts in palliative care using the RI.

$* * * * * * * * *$ 